\title{
Spectral Cauchy Characteristic Extraction of strain, news and gravitational radiation flux
}

\author{
Casey J. Handmer ${ }^{1}$, Béla Szilágyi ${ }^{1,2}$, Jeffrey Winicour ${ }^{3}$ \\ ${ }^{1}$ TAPIR, Walter Burke Institute for Theoretical Physics, MC 350-17, California \\ Institute of Technology, 1200 E California Blvd, Pasadena CA 91125, USA \\ ${ }^{2}$ Jet Propulsion Laboratory, California Institute of Technology, 4800 Oak Grove \\ Dr. Pasadena CA, 91109, USA \\ ${ }^{3}$ Department of Physics and Astronomy University of Pittsburgh, Pittsburgh, PA \\ 15260, USA \\ E-mail: chandmer@caltech.edu
}

\begin{abstract}
.
We present a new approach for the Cauchy-characteristic extraction of gravitational radiation strain, news function, and the flux of the energy-momentum, supermomentum and angular momentum associated with the Bondi-MetznerSachs asymptotic symmetries. In Cauchy-characteristic extraction, a characteristic evolution code takes numerical data on an inner worldtube supplied by a Cauchy evolution code, and propagates it outwards to obtain the space-time metric in a neighborhood of null infinity. The metric is first determined in a scrambled form in terms of coordinates determined by the Cauchy formalism. In prior treatments, the waveform is first extracted from this metric and then transformed into an asymptotic inertial coordinate system. This procedure provides the physically proper description of the waveform and the radiated energy but it does not generalize to determine the flux of angular momentum or supermomentum. Here we formulate and implement a new approach which transforms the full metric into an asymptotic inertial frame and provides a uniform treatment of all the radiation
\end{abstract}


fluxes associated with the asymptotic symmetries. Computations are performed and calibrated using the Spectral Einstein Code (SpEC).

PACS numbers: 04.20Ex, 04.25Dm, 04.25Nx, 04.70Bw 


\section{Introduction}

The strong gravitational radiation produced in the inspiral and merger of binary black holes has been a dominant motivation for the construction of gravitational wave observatories. This effort has recently been brought to fruition by the observation of a binary inspiral and merger by the LIGO gravitational wave detectors [1]. The details of the gravitational waveform supplied by numerical simulation is a key theoretical tool to fully complement the sensitivity of the LIGO and Virgo observatories [2, 3, 4, 5], by enhancing the detection and the scientific interpretation of the gravitational signal. Besides the gravitational waveform, the flux of energymomentum carried off by the waves has important astrophysical effects on the binary system. In particular, the recoil "kick" on the binary due the radiative loss of momentum can possibly eject the final black hole from a galactic center. The strength of such kicks has been computed by various means [6, 7, 8, 9, 10, 11]. The most unambiguous and accurate approach is in terms of the Bondi news function [12], which supplies the gravitational energy and momentum flux at future null infinity $\mathscr{I}^{+}$.

This can be carried out via Cauchy-characteristic extraction (CCE), in which the Cauchy evolution is used to supply the boundary data on a timelike inner worldtube, which then allows a characteristic evolution extending to $\mathscr{I}^{+}$where the radiation is computed using the geometric methods developed by Bondi et al. [12], Sachs [13] and Penrose [14]. For a review, see [15]. A version of this initial-boundary value problem based upon a timelike worldtube [16] has been implemented as a characteristic evolution code, the PITT null code [17, 18, 19, 20], and more recently as the SpEC characteristic code [21, 22], both of which incorporate a Penrose compactification of the exterior space-time extending to $\mathscr{I}^{+}$. In this way, characteristic evolution 
coupled to Cauchy evolution has been implemented to give an accurate numerical computation of the Bondi news function, which determines both the waveform and the radiated energy-momentum.

One technical complication introduced by CCE is that the coordinates induced on $\mathscr{I}^{+}$are related to the Cauchy coordinates on the inner worldtube. Consequently, these computational coordinates do not correspond to inertial observers at $\mathscr{I}^{+}$, i.e. to the coordinates intrinsic to a distant freely falling and non-rotating observatory. Thus, the gravitational waveform first obtained in the "computational coordinates" of $\mathrm{CCE}$ is in a scrambled form. Because the news function is an invariant irrespective of coordinate system, the procedure up to now has been to compute it first in the computational coordinates $\$$ It is then unscrambled by constructing the transformation between code coordinates and inertial coordinates on $\mathscr{I}^{+}$, as portrayed in Fig. 1. Likewise, a physically relevant calculation of the radiation waveform must also be referred to such inertial coordinates on $\mathscr{I}^{+}$.

In addition to energy-momentum loss, the gravitational radiation of angular momentum has important consequences for the evolution of a relativistic binary system. For a historic account of attempts at a universally accepted definition of angular momentum for radiating systems in general relativity see [24]. At spatial infinity, reasonable asymptotic conditions establish the Poincaré group as the asymptotic symmetry group. This allows a Poincaré covariant definition of angular momentum in which the translation freedom mixes angular momentum with linear momentum in the standard manner [25]. However, the Bondi-Metzner-Sachs (BMS) asymptotic symmetry group [26] at $\mathscr{I}^{+}$has an infinite supertranslation subgroup. $\ddagger$ More accurately, the news tensor $N_{a b}$ is a gauge invariant field on $\mathscr{I}^{+}$23]. The news function $N=\frac{1}{4} N_{a b} q^{a} q^{b}$ depends upon a choice of complex polarization dyad $q^{a}$, which has gauge freedom. But this freedom can be trivially unwrapped by the construction $N_{a b}=N \bar{q}_{a} \bar{q}_{b}+\bar{N} q_{a} q_{b}$. 
Fortunately, the translations form an invariant subgroup of the BMS group, which leads to an unambiguous definition of energy-momentum. However, although the Lorentz group is a subgroup of the BMS group, the supertranslations lead to a mixing of the associated supermomentum with angular momentum, the physical consequences of which have not been fully explored. For a stationary epoch in the neighborhood of $\mathscr{I}^{+}$, this supertranslation freedom can be removed and the BMS group reduced to the Poincaré group, in which case angular momentum can be well-defined. However, for a system which makes a stationary to stationary transition, the two Poincaré groups obtained at early and late times can be shifted by a supertranslation. Such supertranslation shifts could lead to a distinctly general relativistic mechanism for a system to lose angular momentum. See [27, 28, 29] for discussions. This in fact occurs if the intervening gravitational radiation produces a non-zero gravitational memory effect. A non-zero gravitational radiation memory is equivalent to such a supertranslation shift [30]. In this paper, we develop a unified algorithm for the computation of the gravitational fluxes of energy-momentum, angular momentum and supermomentum to infinity.

There are two distinct approaches for obtaining flux-conserved quantities which form a representation of the BMS asymptotic symmetry group. One approach consists of the BMS linkage integrals $L_{\xi}(\Sigma)$ [16, 31, 29], which for each spherical cross-section $\Sigma$ of $\mathscr{I}^{+}$generalize the Komar integrals [32] for exact symmetries to the case of asymptotic Killing vectors $\xi^{a}$. Associated with the linkage integrals are locally defined fluxes ${ }^{L} F_{\xi}$ whose integral determines the change $L_{\xi}\left(\Sigma_{2}\right)-L_{\xi}\left(\Sigma_{1}\right)$ between two cross-sections. The second approach, originated by Ashetkar and Streubel, is based upon the Hamiltonian phase space of gravitational radiation modes at $\mathscr{I}^{+}[33]$. The Hamiltonian densities generating a BMS transformation also 
geometrically define local fluxes ${ }^{H} F_{\xi}$. In the case of the supertranslations $\alpha^{a}$, Ashetkar and Streubel showed that these Hamiltonian fluxes could be integrated to obtain flux conserved charges $Q_{\alpha}(\Sigma)$. In the case of the BMS time and space translations, $\xi^{a}=\tau^{a}$, the corresponding linkage and Hamiltonian energy-momentum integrals and their fluxes are identical, i.e. $L_{\tau}(\Sigma)=Q_{\tau}(\Sigma)$ and ${ }^{L} F_{\tau}={ }^{H} F_{\tau}$. However, their supermomentum fluxes differ locally. Subsequently, Wald and Zoupas [34] generalized the Hamiltonian approach and obtained flux conserved quantities for all the AshetkarStreubel Hamiltonian fluxes, including angular momentum. They showed that these flux conserved quantities, including angular momentum, were identical to previous expressions proposed by Dray and Streubel [35].

The relation between the linkage and Hamiltonian BMS fluxes has been examined in [36]. Although their construction and their local values are completely different, it has been shown that the integrals of all the linkage and Hamiltonian fluxes between cross-sections of $\mathscr{I}^{+}$agree, including the angular momentum and supermomentum fluxes, in the most physically relevant case when $\Sigma_{2}$ and $\Sigma_{1}$ are shear-free crosssections in the limits of infinite future and past retarded time, respectively. Although, for the rotations and boosts a factor-of-two anomaly in the linkages must be taken into account, i.e. $\int_{\Sigma}{ }^{L} F_{\xi} d S=2 \int_{\Sigma}{ }^{H} F_{\xi} d S$.

There have been other approaches to defining energy-momentum and angular momentum at $\mathscr{I}^{+}$which do not appeal to the BMS symmetries. Some [35, 37] have been based upon an asymptotic version of Penrose's construction of quasi-local energy-momentum and angular momentum using twistor theory [38]. Another has been based upon the modification of the BMS group to a quasigroup of asymptotic symmetries [39]. Other important work on the computation of the physical properties of radiation at $\mathscr{I}^{+}$has been presented in [40, 41, 42, 43]. 
Here, we concentrate on the linkage approach because it is readily adapted to previous computational infrastructure treating $\mathscr{I}^{+}$. However, the computational methods presented here should also be useful in computing the Hamiltonian charges and fluxes, as formulated by Wald and Zoupas, which have all the desired physical properties. Although the linkage approach is geometrically well defined, its main physical shortcoming is that the associated supermomentum fluxes do not locally vanish in Minkowksi space, although their integrals between cross-sections of $\mathscr{I}^{+}$ determined by two Minkowski space light cones do vanish. As a result, the local physical significance of these supermomentum fluxes is unclear, although the local time rate of change of all the linkage fluxes ${ }^{L} \dot{F}_{\xi}$ do vanish in Minkowski space.

Furthermore, as shown in Sec. 5, in a general radiating spacetime ${ }^{L} \dot{F}_{\xi}=0$ for all BMS generators in any region where the Newman-Penrose radiative component of the Weyl tensor $\psi_{4}^{0}$ vanishes. In particular, ${ }^{L} \dot{F}_{\xi} \rightarrow 0$ for all BMS symmetries in the infinite retarded time past on $\mathscr{I}^{+}$. It is precisely in this limit that the supertranslation freedom can also be eliminated and a preferred Poincaré subgroup can be identified [27]. As a result, the energy-momentum and angular momentum fluxes ${ }^{L} F_{\xi}$ can be uniquely defined by a retarded time integration of ${ }^{L} \dot{F}_{\xi}$, using the initial value ${ }^{L} F_{\xi}=0$ at $u=-\infty$. Similarly, the energy momentum and angular momentum can also be uniquely defined for such systems by a retarded time integration of ${ }^{L} F_{\xi}$, using their initial values at $u=-\infty$, or at spatial infinity. However, there remains the possibility of carrying out a similar construction at $\mathscr{I}^{+}$ in the infinite retarded time future $u=+\infty$. This leads to the same unresolved issue discussed above for a stationary to stationary transition, i.e. to a net supertranslation shift between the future and past Poincaré groups. The identical supertranslation ambiguity exists in the Hamiltonian description of angular momentum. 
As already discussed, in previous applications of CCE to compute energymomentum loss, the Bondi news function was first computed from the radiation field in the computational coordinates by a gauge invariant method. The news and radiation waveform were then transformed to inertial coordinates on $\mathscr{I}^{+}$. In that approach, it was only necessary to find the 3-dimensional transformation to inertial coordinates intrinsic to $\mathscr{I}^{+}$itself. This procedure is possible because the news function can be defined geometrically, without reference to the BMS symmetries. However, this procedure is not feasible in computing the angular momentum and supermomentum fluxes. Here, we present a unified approach to computing all the BMS fluxes of energy-momentum, angular momentum and supermomentum to infinity by carrying out the transformation to inertial coordinates in a full 4-dimensional neighborhood of $\mathscr{I}^{+}$. We are able to accomplish this by constructing a surprisingly simple transformation between the computational and inertial coordinates. The metric is then transformed to the inertial coordinates, in which the BMS symmetries are readily identified and the corresponding fluxes computed. We formulate a simple computational algorithm for carrying out this transformation.

In this procedure, there remains the freedom of the BMS group in the choice of inertial observers. In special relativistic theories, the corresponding freedom reduces to the translations and Lorentz transformations of the Poincaré group. The BMS supertranslations introduce a gauge freedom in the radiation strain.

\section{Waveforms at $\mathscr{I}^{+}$}

The characteristic formalism is based upon a family of outgoing null hypersurfaces emanating from an inner worldtube and extending to infinity, where they foliate $\mathscr{I}^{+}$ 


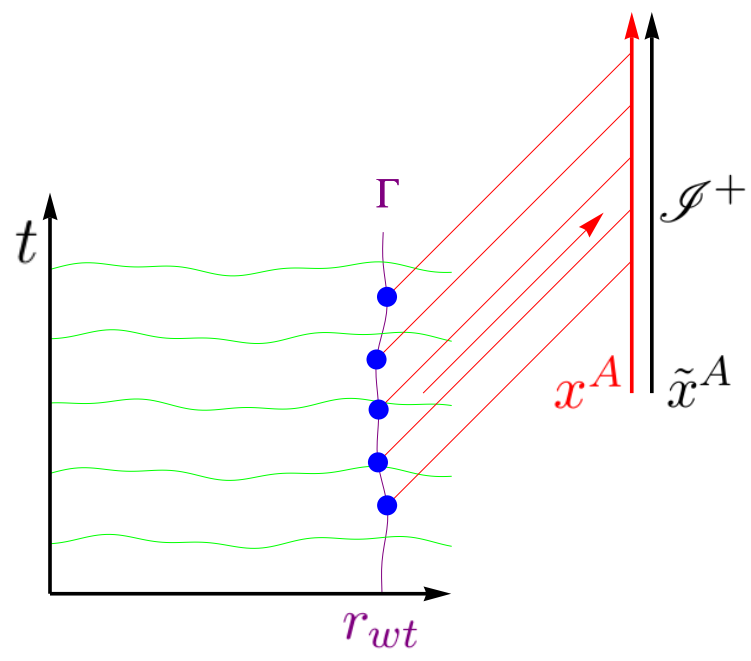

Figure 1: Cauchy characteristic extraction. A Cauchy evolution of the Einstein field equation proceeds on a space-like foliation (green). A finite topologically spherical worldtube $\Gamma$ at areal radius $r_{w t}$ forms the inner boundary to a characteristic evolution on a null foliation (red). Based on a spherical coordinate system $x^{A}$ constructed from Cauchy coordinates on the worldtube, gravitational information is propagated to compactified future null infinity $\mathscr{I}^{+}$. At $\mathscr{I}^{+}$, an inertial coordinate system $\tilde{x}^{A}$ is co-evolved, in which the desired physical waveform can be expressed.

into spherical slices. Let $u$ label these null hypersurfaces, $x^{A}(A=2,3)$ be angular coordinates which label the null rays and $r$ be a surface area coordinate. Using the notion of [16], in the resulting $\left(u, r, x^{A}\right)$ coordinates, the metric takes the Bondi-Sachs form

$$
\begin{aligned}
d s^{2} & =-\left(e^{2 \beta} \frac{V}{r}-r^{2} h_{A B} U^{A} U^{B}\right) d u^{2}-2 e^{2 \beta} d u d r-2 r^{2} h_{A B} U^{B} d u d x^{A} \\
& +r^{2} h_{A B} d x^{A} d x^{B}
\end{aligned}
$$

where $h^{A B} h_{B C}=\delta_{C}^{A}$ and $\operatorname{det}\left(h_{A B}\right)=\operatorname{det}\left(q_{A B}\right)$, with $q_{A B}$ a unit sphere metric.

As described in more detail in [16, 44, in this formalism Einstein's equations decompose into a system which propagate boundary data for the metric variables $\left(\beta, V, U^{A}, h_{A B}\right)$ on an inner worldtube to a solution at $\mathscr{I}^{+}$. In the Pitt null code and in 
the SpEC characteristic code, this solution is computed in a Penrose compactification of $\mathscr{I}^{+}$, e.g. in terms of the coordinates $x^{\mu}=\left(u, \ell, x^{A}\right)$, where $\ell=1 / r, \ell=0$ at $\mathscr{I}^{+}$. Then the conformal metric $\hat{g}_{\mu \nu}=\ell^{2} g_{\mu \nu}$ is smooth at $\mathscr{I}^{+}$and takes the form $\hat{g}_{\mu \nu} d x^{\mu} d x^{\nu}=-\left(\ell^{3} e^{2 \beta} V-h_{A B} U^{A} U^{B}\right) d u^{2}+2 e^{2 \beta} d u d \ell-2 h_{A B} U^{B} d u d x^{A}+h_{A B} d x^{A} d x^{B}$.

General conditions on the asymptotic behavior of the metric variables follow from the vacuum Einstein equations [16],

$$
\begin{gathered}
\beta=H+O\left(\ell^{2}\right), \\
U^{A}=L^{A}+2 \ell e^{2 H} H^{A B} D_{B} H+O\left(\ell^{2}\right), \\
\ell^{2} V=D_{A} L^{A}+\ell\left(e^{2 H} \mathcal{R} / 2+D_{A} D^{A} e^{2 H}\right)+O\left(\ell^{2}\right),
\end{gathered}
$$

and

$$
h_{A B}=H_{A B}+\ell c_{A B}+O\left(\ell^{2}\right)
$$

where $\mathcal{R}$ and $D_{A}$ are the 2-dimensional curvature scalar and covariant derivative associated with $H_{A B}$ and the determinant condition implies

$$
H^{A B} c_{A B}=0
$$

The expansion coefficients $H, H_{A B}, c_{A B}$ and $L^{A}$ (all functions of $u$ and $x^{A}$ ) completely determine the radiation field.

One can further specialize the Bondi coordinates to be inertial at $\mathscr{I}^{+}$, i.e. have asymptotic Minkowski form, in which case $H=L^{A}=0,\left.H_{A B}\right|_{\mathscr{I}+}=q_{A B}$ (the unit sphere metric) so that the radiation field is completely determined by $c_{A B}$, which describes the asymptotic shear of the outgoing null cones or, equivalently, the radiation strain. In these inertial coordinates, the retarded time derivative $\partial_{u} c_{A B}$ 
determines the Bondi news function $N\left(u, x^{A}\right)$. However, the characteristic extraction of the waveform is carried out in computational coordinates derived from the Cauchy coordinates on the inner worldtube, so this inertial simplification cannot be assumed.

In previous work the Bondi news function $N$ was first computed in the computational $\hat{g}_{\mu \nu}$ frame. It was then transformed to inertial coordinates $\left(\tilde{u}, \tilde{x}^{A}\right)$ on $\mathscr{I}^{+}$to determine the physical dependence of the waveform on retarded time and angle. Here we construct the transformation to a compactified version of inertial coordinates $\left(\tilde{u}, \tilde{\ell}, \tilde{x}^{A}\right)$ in a full neighborhood of $\mathscr{I}^{+}$.

\section{Construction of inertial coordinates}

First, we recall some basic elements of Penrose compactification. In a spacetime with metric $g_{\mu \nu}$, the vacuum Einstein equations $G_{\mu \nu}=0$ expressed in terms of a conformally related metric $\hat{g}_{\mu \nu}=\Omega^{2} g_{\mu \nu}$, where $\Omega=0$ on $\mathscr{I}^{+}$, take the form

$$
\Omega^{2} \hat{G}_{\mu \nu}+2 \Omega \hat{\nabla}_{\mu} \hat{\nabla}_{\nu} \Omega-\hat{g}_{\mu \nu}\left(2 \Omega \hat{\nabla}^{\rho} \hat{\nabla}_{\rho} \Omega-3\left(\hat{\nabla}^{\rho} \Omega \hat{\nabla}_{\rho} \Omega\right)\right)=0
$$

It immediately follows that

$$
\left.\left(\hat{\nabla}^{\rho} \Omega\right) \hat{\nabla}_{\rho} \Omega\right|_{\mathscr{I}^{+}}=0
$$

so that $\mathscr{I}^{+}$is a null hypersurface and that

$$
\left.\left[\hat{\nabla}_{\mu} \hat{\nabla}_{\nu} \Omega-\frac{1}{4} \hat{g}_{\mu \nu} \hat{\nabla}^{\rho} \hat{\nabla}_{\rho} \Omega\right]\right|_{\mathscr{I}+}=0
$$

With respect to this general frame, we now choose $\Omega=\ell$ and computational coordinates $\left(u, \ell, x^{A}\right)$, as in Sec. 2, and proceed to construct an inertial conformal frame as follows. We introduce a new conformal factor $\tilde{\Omega}=\omega \Omega=\omega \ell$, with $\tilde{g}_{\mu \nu}=\omega^{2} \hat{g}_{\mu \nu}$ such that

$$
\tilde{\nabla}^{\rho} \tilde{\nabla}_{\rho} \tilde{\Omega}_{\mathscr{I}_{+}}=0
$$


by requiring

$$
\left.\left[2 \hat{n}^{\sigma} \partial_{\sigma} \omega+\omega \hat{\nabla}_{\sigma} \hat{n}^{\sigma}\right]\right|_{\mathscr{I}+}=0, \quad \hat{n}^{\sigma}=\hat{g}^{\rho \sigma} \nabla_{\rho} \ell .
$$

It then follows that

$$
\left.\tilde{\nabla}_{\mu} \tilde{\nabla}_{\nu} \tilde{\Omega}\right|_{\mathscr{I}^{+}}=0,\left.\quad \tilde{\nabla}^{\rho} \tilde{\nabla}_{\rho} \tilde{\Omega}\right|_{\mathscr{I}^{+}}=0,
$$

i.e. in the $\tilde{g}_{\mu \nu}$ conformal frame $\mathscr{I}^{+}$is null, shear-free and divergence-free. It also follows that

$$
\left.\tilde{n}^{\sigma} \tilde{\nabla}_{\sigma} \tilde{n}^{\nu}\right|_{\mathscr{I}+}=0, \quad \tilde{n}^{\sigma}=\tilde{g}^{\rho \sigma} \tilde{\nabla}_{\rho} \tilde{\Omega},
$$

i.e. in the $\tilde{g}_{\mu \nu}$ frame $\tilde{n}^{\sigma}$ is an affinely parametrized null generator of $\mathscr{I}^{+}$.

We now construct inertial conformal coordinates $\left(\tilde{u}, \tilde{x}^{A}\right)$ on $\mathscr{I}^{+}$, by first assigning angular coordinates $\tilde{x}^{A}$ to each point of some initial spacelike spherical slice $\tilde{u}=u_{0}$ of $\mathscr{I}^{+}$. We then propagate these coordinates along the null geodesics generating $\mathscr{I}^{+}$ according to

$$
\left.\tilde{n}^{\rho} \partial_{\rho} \tilde{x}^{A}\right|_{\mathscr{I}^{+}}=\left.\omega^{-1} \hat{n}^{\rho} \partial_{\rho} \tilde{x}^{A}\right|_{\mathscr{I}^{+}}=0 .
$$

In addition, we require

$$
\left.\tilde{n}^{\rho} \partial_{\rho} \tilde{u}\right|_{\mathscr{I}^{+}}=\left.\omega^{-1} \hat{n}^{\rho} \partial_{\rho} \tilde{u}\right|_{\mathscr{I}^{+}}=1,
$$

so that $\tilde{u}$ is an affine parameter along the generators in the $\tilde{g}_{\mu \nu}$ conformal frame.

This determines the transformation from the computational coordinates $x^{\mu}=$ $\left(u, \ell, x^{A}\right)$ to inertial coordinates $\left(\tilde{u}\left(u, x^{B}\right), \tilde{x}^{A}\left(u, x^{B}\right)\right)$ on $\mathscr{I}^{+}$, which allows the news function and the extracted waveform to be re-expressed in the physically relevant coordinates of a detector. However, this is not sufficient to identify the BMS symmetries and their associated fluxes. The remaining complication is that after transforming to inertial coordinates the metric on the spherical cross-sections of $\mathscr{I}^{+}$,

$$
H_{\tilde{A} \tilde{B}}=\left.\hat{g}_{\tilde{A} \tilde{B}}\right|_{\mathscr{I}+}=\left.\frac{\partial x^{\mu}}{\partial \tilde{x}^{A}} \frac{\partial x^{\nu}}{\partial \tilde{x}^{B}} \hat{g}_{\mu \nu}\right|_{\mathscr{I}+},
$$


does not reduce to a unit sphere metric. As a result, the identification of the translations as a subgroup of the supertranslation group is complicated; essentially one must solve an elliptic equation to identify the curved 2 -space version of the $\ell=0$ and $\ell=1$ spherical harmonics on the spherical cross-sections.

For this purpose, it is simplest to proceed by determining the conformal factor $\omega$ relating $H_{\tilde{A} \tilde{B}}$ to a unit-sphere metric $Q_{\tilde{A} \tilde{B}}$,

$$
Q_{\tilde{A} \tilde{B}}=\omega^{2} H_{\tilde{A} \tilde{B}}
$$

We determine $\omega$ by solving the elliptic equation governing the conformal transformation of the curvature scalar to the unit sphere curvature,

$$
\mathcal{R}=2\left(\omega^{2}+D_{A} D^{A} \log \omega\right)
$$

where $\mathcal{R}$ and $D_{A}$ are the curvature scalar and covariant derivative associated with the 2-metric $H_{A B}$. Since this is a scalar equation it can be solved in the computational coordinates.

The elliptic equation (3.12) need only be solved at the initial time. Then, the shear-free property of the null geodesics generating $\mathscr{I}^{+}$implies that $\omega$ may be propagated along the generators by means of (3.6), which takes the explicit form

$$
2 \hat{n}^{\alpha} \partial_{\alpha} \log \omega=-e^{-2 H} D_{A} L^{A}
$$

After initialization of $\omega$ so that the initial slice of $\mathscr{I}^{+}$has unit sphere geometry, it then follows that all cross-sections of $\mathscr{I}^{+}$have unit sphere geometry. In terms of standard spherical coordinates $\tilde{x}^{A}=(\tilde{\theta}, \tilde{\phi})$, the induced metric on the cross-sections of $\mathscr{I}^{+}$has components

$$
\left.\tilde{g}_{\tilde{A} \tilde{B}}\left(\tilde{u}, \tilde{x}^{A}\right)\right|_{\mathscr{I}+}=Q_{\tilde{A} \tilde{B}},\left.\quad \tilde{g}^{\tilde{A} \tilde{B}}\left(\tilde{u}, \tilde{x}^{A}\right)\right|_{\mathscr{I}+}=Q^{\tilde{A} \tilde{B}}
$$


where, in these coordinates,

$$
Q_{\tilde{A} \tilde{B}} d \tilde{x}^{A} d \tilde{x}^{B}=d \tilde{\theta}^{2}+\sin ^{2} \tilde{\theta} d \tilde{\phi}^{2}
$$

Given such inertial coordinates on $\mathscr{I}^{+}$, we then extend them to coordinates $\tilde{x}^{\mu}=\left(\tilde{u}, \tilde{\ell}, \tilde{x}^{A}\right)$ in a neighborhood of $\mathscr{I}^{+}$, where

$$
\tilde{\ell}=\omega\left(u, x^{A}\right) \ell=\tilde{\Omega} .
$$

The Jacobian of the transformation from computational to inertial coordinates has the simple property

$$
\partial_{\ell} \tilde{u}=\partial_{\ell} \tilde{x}^{A}=0, \quad \partial_{\ell} \tilde{\ell}=\omega .
$$

As a result it immediately follows that at $\mathscr{I}^{+}$the metric reduces to the simple form

$$
\begin{gathered}
\left.\tilde{g}_{\tilde{A} \tilde{B}}\left(\tilde{u}, \tilde{x}^{A}\right)\right|_{\mathscr{I}+}=Q_{\tilde{A} \tilde{B}},\left.\quad \tilde{g}^{\tilde{A} \tilde{B}}\left(\tilde{u}, \tilde{x}^{A}\right)\right|_{\mathscr{I}+}=Q^{\tilde{A} \tilde{B}}, \\
\left.\tilde{g}_{\tilde{u} \tilde{u}}\left(\tilde{u}, \tilde{x}^{A}\right)\right|_{\mathscr{I}+}=\left.\tilde{g}_{\tilde{u} \tilde{A}}\left(\tilde{u}, \tilde{x}^{A}\right)\right|_{\mathscr{I}+}=\left.\tilde{g}^{\tilde{\ell} \tilde{\ell}}\left(\tilde{u}, \tilde{x}^{A}\right)\right|_{\mathscr{I}+}=\left.\tilde{g}^{\tilde{\ell} \tilde{A}}\left(\tilde{u}, \tilde{x}^{A}\right)\right|_{\mathscr{I}+}=0 .
\end{gathered}
$$

This transformation to inertial coordinates also determines the metric in a neighborhood of $\mathscr{I}^{+}$, which simplifies the identification of the BMS group and the computation of the radiation strain and BMS fluxes.

\section{The BMS group}

In the inertial coordinates and conformal frame constructed in Sec. 3 , the asymptotic Killing vectors composing the BMS group can be described by by [13, 16]

$$
\left.\xi^{\tilde{\rho}} \partial_{\tilde{\rho}}\right|_{\mathscr{I}+}=\left(\alpha\left(\tilde{x}^{A}\right)+\frac{1}{2} \tilde{u} f_{: \tilde{A}}^{\tilde{A}}\right) \partial_{\tilde{u}}+f^{\tilde{A}} \partial_{\tilde{A}},
$$

where a "colon" denotes the covariant derivative with respect to the unit sphere metric

$Q_{\tilde{A} \tilde{B}}, \alpha\left(\tilde{x}^{A}\right)$ represents the supertranslation freedom and $f^{\tilde{A}}\left(\tilde{x}^{B}\right)$ is a conformal Killing vector on the unit sphere,

$$
f^{(\tilde{A}: \tilde{B})}=\frac{1}{2} Q^{\tilde{A} \tilde{B}} f_{: \tilde{C}}^{\tilde{C}}
$$


This conformal group is isomorphic to the Lorentz group.

The additional property that $\xi^{\mu}$ is tangent to $\mathscr{I}^{+}$,

$$
\left.\xi^{\tilde{\ell}}\right|_{\mathscr{I}+}=\left.\xi^{\rho} \partial_{\rho} \tilde{\Omega}\right|_{\mathscr{I}+}=0
$$

implies that $\xi^{\mu}$ satisfies the asymptotic Killing equation in the physical space geometry,

$$
\left.\tilde{\Omega}^{-2} \nabla^{(\mu} \xi^{\nu)}\right|_{\mathscr{I}^{+}}=\left.\left[\tilde{\nabla}^{(\tilde{\mu}} \tilde{\mathcal{\nu}}^{\tilde{\nu})}-\tilde{\Omega}^{-1} \tilde{g}^{\tilde{\mu} \tilde{\nu}} \xi^{\tilde{\rho}} \tilde{\nabla}_{\tilde{\rho}} \tilde{\Omega}\right]\right|_{\mathscr{I}^{+}}=0 .
$$

The supertranslations form an invariant subgroup of the BMS group for which $f^{\tilde{A}}=0$. The translations are an invariant 4-parameter subgroup of the supertranslations for which $\alpha\left(\tilde{x}^{A}\right)$ is composed of $\ell=0$ and $\ell=1$ spherical harmonics. The rotation subgroup intrinsic to a cross-section $\Sigma^{+}$of $\mathscr{I}^{+}$consists of the BMS transformations which map $\Sigma^{+}$onto itself. Without introducing any artificially preferred structure on $\mathscr{I}^{+}$, there is no invariant way to extract a rotation group, Lorentz group or Poincaré group from the BMS group.

Given a cross-section $\Sigma^{+}$of $\mathscr{I}^{+}$and a generator $\xi^{\mu}$ of the BMS group, the linkage integral $L_{\xi}\left(\Sigma^{+}\right)$which generalizes the Komar integral for an exact symmetry is given in terms of the physical space geometry by

$$
L_{\xi}\left(\Sigma^{+}\right)=\lim _{\Sigma \rightarrow \Sigma^{+}} \oint_{\Sigma}\left(\nabla^{[\mu} \xi^{\mu]}-K^{[\mu} N^{\nu]} \nabla_{\rho} \xi^{\rho}\right) d S_{\mu \nu},
$$

where $K^{\mu}$ and $N^{\mu}$ are, respectively, ingoing and outgoing null vectors normal to $\Sigma$, normalized by $K^{\mu} N_{\mu}=-1$. The limit is taken along the outgoing null hypersurface $\mathcal{N}$ emanating from $\Sigma$ to $\Sigma^{+}$, as shown in Fig. 2. The value of this limit depends upon how the BMS generator is extended off $\mathscr{I}^{+}$. The asymptotic symmetry condition (4.4) allows the freedom in this extension of the form

$$
\xi^{\mu} \rightarrow \xi^{\mu}+\tilde{\Omega}^{2} v^{\mu} .
$$




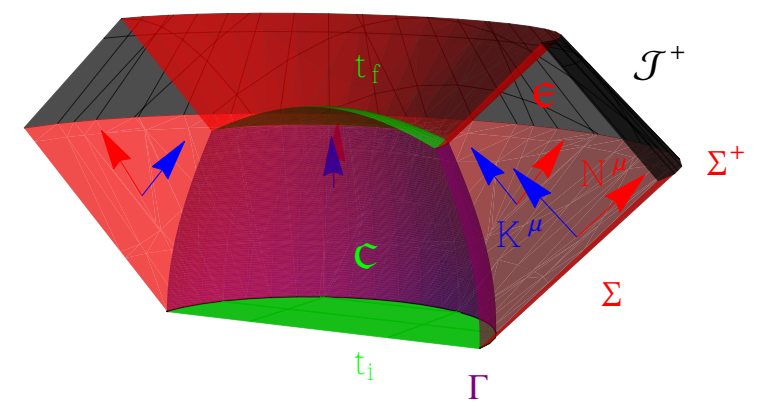

Figure 2: Diagram of the computational domain under Penrose compactification, with angle $\tilde{\theta}$ suppressed. The Cauchy evolution $\mathcal{C}$ runs from time $t_{i}$ to $t_{f}$ within the worldtube $\Gamma$. The characteristic evolution $\mathcal{E}$ is performed on null hypersurfaces extending from $\Gamma$ to future null infinity $\mathscr{I}^{+}$. Cross-sections $\Sigma$ of a null hypersurface (topologically a 2-sphere) approach the limit $\Sigma^{+}$at $\mathscr{I}^{+}$, where the linkage integral is defined. Ingoing $\left(K^{\mu}\right)$ and outgoing $\left(N^{\mu}\right)$ null vectors are normal to $\Sigma$. Gauge independent news, strain, $\psi_{4}^{0}$ and flux are computed in inertial coordinates at $\mathscr{I}^{+}$.

In the original formulation of the linkages [16], $v^{\mu}$ was determined by a null hypersurface propagation law on $\mathcal{N}$,

$$
\left(\nabla^{(\mu} \xi^{\nu)}-\frac{1}{2} g^{\mu \nu} \nabla_{\rho} \xi^{\rho}\right) K_{\mu}=0
$$

In [29], other choices of propagation law were considered. In the next section. we adopt a simple extension in which the generators $\xi^{\mu}$ only depend linearly on the inertial conformal factor $\tilde{\ell}$.

\section{The BMS fluxes}

Our focus is on the radiation flux $F_{\xi}$ across $\mathscr{I}^{+}$which governs the change of linkage between two cross-sections,

$$
L_{\xi}\left(\Sigma_{2}\right)-L_{\xi}\left(\Sigma_{1}\right)=\int_{\Sigma_{1}^{+}}^{\Sigma_{2}^{+}} F_{\xi} d V
$$


In a conformal frame in which $\mathscr{I}^{+}$is divergence-free, it was shown in [29] that there is a local geometric expression for $F_{\xi}$ which is independent of the freedom 4.6) governing the extension of $\xi^{\mu}$ off $\mathscr{I}^{+}$. The resulting flux is uniquely determined by (i) the choice of $\xi^{\mu}$ on $\mathscr{I}^{+}$and (ii) the local conformal geometry near $\mathscr{I}^{+}$; and (iii) it is independent of any choice of cross-section or other extraneous constructs.

In the $\tilde{g}_{\tilde{\mu} \tilde{\nu}}$ conformal inertial frame described in Sec. 3, this flux is constructed as follows. The transformation from the computational coordinates $x^{\mu}\left(u, \ell, x^{A}\right)$ to inertial coordinates $\left(\tilde{u}\left(u, x^{B}\right), \tilde{x}^{A}\left(u, x^{B}\right)\right)$ on $\mathscr{I}^{+}$is first extended to coordinates $\tilde{x}^{\mu}=\left(\tilde{u}, \tilde{\ell}, \tilde{x}^{A}\right)$ in a neighborhood of $\mathscr{I}^{+}$where, as before,

$$
\tilde{\ell}=\omega\left(u, x^{A}\right) \ell=\tilde{\Omega} .
$$

This transformation determines the metric in the extension of the inertial frame to a neighborhood of $\mathscr{I}^{+}$,

$$
\tilde{g}^{\tilde{\mu} \tilde{\nu}}\left(\tilde{u}, \tilde{\ell}, \tilde{x}^{A}\right)=\omega^{-2} \frac{\partial \tilde{x}^{\mu}}{\partial x^{\alpha}} \frac{\partial \tilde{x}^{\nu}}{\partial x^{\beta}} \hat{g}^{\alpha \beta}\left(u, \ell, x^{A}\right) .
$$

In addition to (3.18) and (3.19), this implies

$$
\begin{aligned}
& \tilde{g}^{\tilde{u} \tilde{\ell}}\left(\tilde{u}, 0, \tilde{x}^{A}\right)=1, \\
& \tilde{g}^{\tilde{u} \tilde{u}}\left(\tilde{u}, 0, \tilde{x}^{A}\right)=\omega^{-2} \frac{\partial \tilde{u}}{\partial x^{A}} \frac{\partial \tilde{u}}{\partial x^{B}} \hat{g}^{A B}, \\
& \tilde{g}^{\tilde{u} \tilde{A}}\left(\tilde{u}, 0, \tilde{x}^{A}\right)=\omega^{-2} \frac{\partial \tilde{u}}{\partial x^{B}} \frac{\partial \tilde{x}^{A}}{\partial x^{C}} \hat{g}^{B C} .
\end{aligned}
$$

The corresponding covariant components are

$$
\begin{aligned}
& \tilde{g}_{\tilde{u} \tilde{\ell}}\left(\tilde{u}, 0, \tilde{x}^{A}\right)=1 \\
& \tilde{g}_{\tilde{\ell} \tilde{\ell}}\left(\tilde{u}, 0, \tilde{x}^{A}\right)=-\tilde{g}^{\tilde{u} \tilde{u}}+Q_{\tilde{A} \tilde{B}} \tilde{g}^{\tilde{u} \tilde{A}} \tilde{g}^{\tilde{u} \tilde{B}}, \\
& \tilde{g}_{\tilde{\ell} \tilde{A}}\left(\tilde{u}, 0, \tilde{x}^{A}\right)=-Q_{\tilde{A} \tilde{B}} \tilde{g}^{\tilde{u} \tilde{B}} .
\end{aligned}
$$

Note that the $\tilde{x}^{\mu}$ coordinates are not null coordinates. While the $\tilde{u}=$ const crosssections of $\mathscr{I}^{+}$are space-like, 5.4 implies in general that $\tilde{g}^{\tilde{u} \tilde{u}}>0$ so that the 
$\tilde{u}=$ const hypersurfaces in the neighborhood of $\mathscr{I}^{+}$are asymptotically time-like. This somewhat surprising feature stems from the requirement that the transformation from computational coordinates to inertial coordinates is linear in $\ell$. The transformation to the $\tilde{x}^{\mu}$ inertial coordinates determines the essential geometric quantities associated with the inertial frame metric $\tilde{g}_{\tilde{\mu} \tilde{\nu}}$, e.g. the associated covariant derivative $\tilde{\nabla}_{\tilde{\mu}}$ and curvature scalar $\tilde{R}$.

Restrictions on the $\tilde{\ell}$-derivatives of the inertial metric at $\mathscr{I}^{+}$arise from 3.6 ,

$$
\begin{aligned}
& \left.\partial_{\tilde{\ell}} \tilde{g}_{\tilde{u} \tilde{u}}\right|_{\mathscr{I}+}=0, \\
& \left.\partial_{\tilde{\ell}} \tilde{g}_{\tilde{u} \tilde{\ell}}\right|_{\mathscr{I}+}=\left.\frac{1}{2} \partial_{\tilde{u}} \tilde{g}_{\tilde{\ell} \tilde{\ell}}\right|_{\mathscr{I}+}, \\
& \left.\partial_{\tilde{\ell}} \tilde{g}_{\tilde{u} \tilde{A}}\right|_{\mathscr{I}+}=\left.\partial_{\tilde{u}} \tilde{g}_{\tilde{A} \tilde{\ell}}\right|_{\mathscr{I}+} .
\end{aligned}
$$

In addition,

$$
\tilde{g}_{\tilde{u} \tilde{\ell}}=\frac{\partial u}{\partial \tilde{u}} \omega e^{2 \beta}
$$

so since $(2.3)$ implies $\left.\partial_{\tilde{\ell}} \beta\right|_{\mathscr{I}+}=0$, we have

$$
\left.\partial_{\tilde{\ell}} \tilde{g}_{\tilde{u} \tilde{\ell}}\right|_{\mathscr{I}+}=\left.\partial_{\tilde{u}} \tilde{g}_{\tilde{\ell} \tilde{\ell}}\right|_{\mathscr{I}+}=0 \text {. }
$$

The determinant condition (2.7), i.e. $\left.h^{A B} \partial_{\ell} h_{A B}\right|_{\mathscr{I}^{+}}=0$ implies

$$
\left.\left\{Q^{\tilde{A} \tilde{B}} \partial_{\tilde{\ell}} \tilde{g}_{\tilde{A} \tilde{B}}+2 \tilde{g}^{\tilde{u} \tilde{A}} \partial_{\tilde{\ell}} \tilde{g}_{\tilde{u} \tilde{A}}\right\}\right|_{\mathscr{I}+}=0
$$

so that

$$
\left.\left\{Q^{\tilde{A} \tilde{B}} \partial_{\tilde{\ell}} \tilde{g}_{\tilde{A} \tilde{B}}+2 \tilde{g}^{\tilde{u} \tilde{A}} \partial_{\tilde{u}} \tilde{g}_{\tilde{\ell} \tilde{A}}\right\}\right|_{\mathscr{I}+}=\left.\left\{Q^{\tilde{A} \tilde{B}} \partial_{\tilde{\ell}} \tilde{g}_{\tilde{A} \tilde{B}}-2 Q_{\tilde{A} \tilde{B}} \tilde{g}^{\tilde{u} \tilde{A}} \partial_{\tilde{u}} \tilde{g}^{\tilde{u} \tilde{B}}\right\}\right|_{\mathscr{I}+}=0 .
$$

The corresponding contravariant components satisfy

$$
\begin{aligned}
\partial_{\tilde{\ell}} \tilde{g}^{\left.\tilde{\ell}\right|_{\mathscr{I}+}} & =0, \\
\left.\partial_{\tilde{\ell}} \tilde{g}^{\tilde{\ell} \tilde{A}}\right|_{\mathscr{I}+} & =\left.\partial_{\tilde{u}} \tilde{g}^{\tilde{u} \tilde{A}}\right|_{\mathscr{I}+}, \\
\left.\partial_{\tilde{\ell}} \tilde{u^{\tilde{\ell}}}\right|_{\mathscr{I}+}=\left.\frac{1}{2} \partial_{\tilde{u}} \tilde{g}^{\tilde{u} \tilde{u}}\right|_{\mathscr{I}+} & =\left.Q_{\tilde{A} \tilde{B}} g^{\tilde{u} \tilde{A}} \partial_{\tilde{u}} \tilde{g}^{\tilde{u} \tilde{B}}\right|_{\mathscr{I}+}, \\
\left.Q_{\tilde{A} \tilde{B}} \partial_{\tilde{\ell}} \tilde{g}^{\tilde{A} \tilde{B}}\right|_{\mathscr{I}+} & =0 .
\end{aligned}
$$


In general, since $\xi^{\tilde{\mu}}$ is tangent to $\mathscr{I}^{+}$, we can define a smooth scalar field $K=\tilde{\Omega}^{-1} \xi^{\tilde{\mu}} \partial_{\tilde{\mu}} \tilde{\Omega}$ and the asymptotic Killing equation 4.4 implies we can define a smooth field $X^{\tilde{\mu} \tilde{\nu}}$ according to

$$
\tilde{\nabla}^{(\tilde{\mu}} \xi^{\tilde{\nu})}=K \tilde{g}^{\tilde{\mu} \tilde{\nu}}+\tilde{\Omega} X^{\tilde{\mu} \tilde{\nu}} .
$$

We write $X=X_{\tilde{\mu}}^{\tilde{\mu}}$. In the $\tilde{g}_{\tilde{\mu} \tilde{\nu}}$ inertial frame,

$$
K=\tilde{\ell}^{-1} \xi^{\tilde{\ell}},
$$

so that

$$
\left.K\right|_{\mathscr{I}^{+}}=\left.\frac{\partial \xi^{\tilde{\ell}}}{\partial \tilde{\ell}}\right|_{\mathscr{I}+} .
$$

Evaluation of (5.12) at $\mathscr{I}^{+}$gives, in addition to (4.1),

$$
\begin{aligned}
& \left.\frac{\partial \xi^{\tilde{\ell}}}{\partial \tilde{\ell}}\right|_{\mathscr{I}+}=\left.\frac{\partial \xi^{\tilde{u}}}{\partial \tilde{u}}\right|_{\mathscr{I}^{+}}=\left.\frac{1}{2} \xi_{: \tilde{A}}^{\tilde{A}}\right|_{\mathscr{I}+}, \\
& \left.\frac{\partial \xi^{\tilde{u}}}{\partial \tilde{\ell}}\right|_{\mathscr{I}+}=\left.\left\{-\tilde{g} \frac{\partial \tilde{u} \tilde{A}}{\partial x^{\tilde{A}}}+\frac{1}{2} \xi^{\tilde{u}} \frac{\partial \tilde{g}^{\tilde{u} \tilde{u}}}{\partial \tilde{u}}+\frac{1}{2} \xi^{\tilde{A}} \frac{\partial \tilde{g}^{\tilde{u} \tilde{u}}}{\partial x^{\tilde{A}}}\right\}\right|_{\mathscr{I}+}, \\
& \left.\frac{\partial \xi^{\tilde{A}}}{\partial \tilde{\ell}}\right|_{\mathscr{I}_{+}}=\left.\left\{\tilde{g}^{\tilde{A} \tilde{u}} \frac{\partial \xi^{\tilde{u}}}{\partial \tilde{u}}-\tilde{g}^{\tilde{u} \tilde{B}} \xi_{: \tilde{B}}^{\tilde{A}}+\xi^{\tilde{B}} g^{\tilde{u} \tilde{A}}: \tilde{B}+\xi^{\tilde{u}} \frac{\partial \tilde{g}^{\tilde{u} \tilde{A}}}{\partial \tilde{u}}-Q^{\tilde{A} \tilde{B}} \frac{\partial \xi^{\tilde{u}}}{\partial x^{\tilde{B}}}\right\}\right|_{\mathscr{I}+} .
\end{aligned}
$$

As shown in [29], it also follows that

$$
\left.X^{\tilde{\mu} \tilde{\nu}} \partial_{\tilde{\nu}} \tilde{\Omega}\right|_{\mathscr{I}_{+}}=0,
$$

which results from a straightforward calculation using (3.19), (5.13) and (5.15). Thus we can further define a smooth field $X^{\tilde{\mu}}$ according to

$$
X^{\tilde{\mu}}=\tilde{\Omega}^{-1} X^{\tilde{\mu} \tilde{\nu}} \partial_{\tilde{\nu}} \tilde{\Omega} .
$$

Let $Q^{\tilde{A}}\left(\tilde{x}^{B}\right)$ be a complex polarization dyad satisfying

$$
Q^{(\tilde{A}} \bar{Q}^{\tilde{B})}=Q^{\tilde{A} \tilde{B}}
$$

We have

$$
\left.Q^{\tilde{A}} Q^{\tilde{B}} \partial_{\tilde{\ell}} \tilde{g}_{\tilde{A} \tilde{B}}\right|_{\mathscr{I}+}=-\left.Q_{\tilde{A}} Q_{\tilde{B}}\left\{\partial_{\tilde{\ell}} \tilde{g}^{\tilde{A} \tilde{B}}-2 \tilde{g}^{\tilde{u} \tilde{A}} \partial_{\tilde{u}} \tilde{g}^{\tilde{u} \tilde{B}}\right\}\right|_{\mathscr{I}+}
$$


The outgoing null vector normal to the $(\tilde{u}=$ const, $\tilde{\ell}=$ const $) 2$-surfaces is

$$
N_{\tilde{\alpha}}=\partial_{\tilde{\alpha}} \tilde{u}-\frac{1}{2} \tilde{g}^{\tilde{u} \tilde{u}} \partial_{\tilde{\alpha}} \tilde{\ell}
$$

The shear or asymptotic strain $h$ on a constant inertial time cross-section of $\mathscr{I}^{+}$is then given by

$$
h=\left.\frac{1}{2} Q^{\tilde{A}} Q^{\tilde{B}} \tilde{\nabla}_{\tilde{A}} N_{\tilde{B}}\right|_{\mathscr{I}+}
$$

so that

$$
\begin{aligned}
h & =\left.\frac{1}{4} Q^{\tilde{A}} Q^{\tilde{B}}\left\{\partial_{\tilde{\ell}} \tilde{g}_{\tilde{A} \tilde{B}}-2 \tilde{g}_{\tilde{\ell} \tilde{A}: \tilde{B}}\right\}\right|_{\mathscr{I}+}, \\
& =\left.\frac{1}{4} Q_{\tilde{A}} Q_{\tilde{B}}\left\{2 \tilde{g}^{\tilde{A} \tilde{u}} \partial_{\tilde{u}} \tilde{g}^{\tilde{B} \tilde{u}}+2 \tilde{g}^{\tilde{u} \tilde{A}: \tilde{B}}-\partial_{\tilde{\ell}} \tilde{g}^{\tilde{A} \tilde{B}}\right\}\right|_{\mathscr{I}+} .
\end{aligned}
$$

Here,

$$
\partial_{\tilde{\ell}} \tilde{g}^{\tilde{A} \tilde{B}}=\omega^{-2} x^{\tilde{A}},{ }_{C} x^{\tilde{B}}{ }_{D} \ell, \tilde{\ell}_{\ell} \partial_{\ell} \hat{g}^{C D}=\omega^{-3} x^{\tilde{A}}{ }_{C} x^{\tilde{B}}{ }_{D} \partial_{\ell} \hat{g}^{C D}
$$

The strain $h$ has gauge freedom corresponding to the supertranslation freedom in the choice of slicing of $\mathscr{I}^{+}$. A gauge independent description of the radiation waveform is given by the news function

$$
N=\left.\frac{1}{2} Q_{\tilde{A}} Q_{\tilde{B}} X^{\tilde{A} \tilde{B}}\right|_{\mathscr{I}+}=\partial_{\tilde{u}} h
$$

where $X^{\tilde{A} \tilde{B}}$ corresponds to the translation

$$
\left.\xi^{\tilde{\alpha}}\right|_{\mathscr{I}^{+}}=\left.\tilde{\nabla}^{\tilde{\alpha}} \tilde{\ell}\right|_{\mathscr{I}+}=(1,0,0,0)
$$

with

$$
\left.\partial_{\tilde{\ell}} \xi^{\tilde{\alpha}}\right|_{\mathscr{I}+}=\left.\partial_{\tilde{u}}\left(\frac{1}{2} \tilde{g}^{\tilde{u} \tilde{u}}, 0, \tilde{g}^{\tilde{u} \tilde{A}}\right)\right|_{\mathscr{I}+}
$$

A short calculation gives

$$
N=\frac{1}{4} Q_{\tilde{A}} Q_{\tilde{B}} \partial_{\tilde{u}}\left(2 \tilde{g}^{\tilde{u} \tilde{A}: \tilde{B}}+2 \tilde{g}^{\tilde{u} \tilde{A}} \partial_{\tilde{u}} \tilde{g}^{\tilde{u} \tilde{B}}-\partial_{\tilde{\ell}} \tilde{g}^{\tilde{A} \tilde{B}}\right)
$$


in accordance with 5.23 and $(5.25)$.

The absolute square of the news function determines the flux of energy and momentum. The flux corresponding to a general asymptotic symmetry is given by [29]

$$
F_{\xi}=-\tilde{\nabla}_{\tilde{\mu}} \tilde{\nabla}_{\tilde{\nu}} X^{\tilde{\mu} \tilde{\nu}}+3 \tilde{\nabla}_{\tilde{\mu}} X^{\tilde{\mu}}+\frac{3}{4} \tilde{\nabla}_{\tilde{\mu}} \tilde{\nabla}^{\tilde{\mu}} X+\frac{1}{24} \tilde{R} X
$$

Note that $F_{\xi}$ is a scalar so that it could be evaluated in any coordinate system. However, its physical properties are only manifest in the inertial $\tilde{x}^{\mu}$ coordinates.

Since $F_{\xi}$ is independent of the freedom (4.6), it suffices to extend the BMS generators to a neighborhood of $\mathscr{I}^{+}$with the linear $\tilde{\ell}$-dependence

$$
\xi^{\tilde{\mu}}\left(\tilde{u}, \tilde{\ell}, \tilde{x}^{A}\right)=\xi^{\tilde{\mu}}\left(\tilde{u}, 0, \tilde{x}^{A}\right)+\tilde{\ell} \frac{\partial \xi^{\tilde{\mu}}}{\partial \tilde{\ell}}\left(\tilde{u}, 0, \tilde{x}^{A}\right)
$$

where the coefficients are determined by (4.1) and (5.15). This determines the asymptotic Killing vectors in the neighborhood of $\mathscr{I}^{+}$for the purpose of computing the flux in terms of 5.29 .

It is also possible to compute the time derivative of the flux in terms of its relation to the Weyl tensor [29],

$$
\dot{F}_{\xi}:=\tilde{n}^{\mu} \partial_{\mu} F_{\xi}=-\left.\Omega^{-1} \tilde{C}_{\alpha \beta \gamma \delta} \tilde{n}^{\beta} \tilde{n}^{\delta} X^{\alpha \gamma}\right|_{\mathscr{I}^{+}}
$$

where asymptotic flatness implies $C_{\alpha \beta \gamma \delta}=O(\Omega)$. In inertial coordinates, in which $\left.X^{\tilde{\alpha} \tilde{\ell}}\right|_{\mathscr{I}+}=0$, this reduces to

$$
\dot{F}_{\xi}=\partial_{\tilde{u}} F_{\xi}=-\left.\partial_{\tilde{\ell}} \tilde{C}_{\tilde{A} \tilde{u} \tilde{B} \tilde{u}} X^{\tilde{A} \tilde{B}}\right|_{\mathscr{I}+}
$$

By virtue of the trace-free property of the Weyl tensor this may be rewritten

$$
\partial_{\tilde{u}} F_{\xi}=-\left.\frac{1}{4}\left\{\partial_{\tilde{\ell}} \tilde{C}_{\tilde{A} \tilde{u} \tilde{B} \tilde{u}} Q^{\tilde{A}} Q^{\tilde{B}} X^{\tilde{C} \tilde{D}} \bar{Q}_{\tilde{C}} \bar{Q}_{\tilde{D}}+\partial_{\tilde{\ell}} \tilde{C}_{\tilde{A} \tilde{u} \tilde{B} \tilde{u}} \bar{Q}^{\tilde{A}} \bar{Q}^{\tilde{B}} X^{\tilde{C} \tilde{D}} Q_{\tilde{C}} Q_{\tilde{D}}\right\}\right|_{\mathscr{I}+}
$$

or

$$
\partial_{\tilde{u}} F_{\xi}=\Psi \overline{\mathcal{X}}+\bar{\Psi} \mathcal{X}
$$


where $\mathcal{X}=\left.\frac{1}{2} X^{\tilde{A} \tilde{B}} Q_{\tilde{A}} Q_{\tilde{B}}\right|_{\mathscr{I}+}$ and $\Psi=-\left.\frac{1}{2} \partial_{\tilde{\ell}} \tilde{C}_{\tilde{A} \tilde{u} \tilde{B} \tilde{u}} Q^{\tilde{A}} Q^{\tilde{B}}\right|_{\mathscr{I}+}$. In Newman-Penrose notation $\bar{\Psi}$ is the asymptotic $\psi_{4}^{0}$ component of the Weyl tensor.

A straightforward computation based upon $(5.12)$ gives

$$
\begin{aligned}
\mathcal{X} & =\left.\frac{1}{2} X^{\tilde{A} \tilde{B}} Q_{\tilde{A}} Q_{\tilde{B}}\right|_{\mathscr{I}+} \\
& =\frac{1}{4} Q_{\tilde{A}} Q_{\tilde{B}}\left\{2\left(\partial_{\tilde{\ell}} \tilde{g}^{\tilde{A} \tilde{C}}\right) \xi^{\tilde{B}}: \tilde{C}\right. \\
& \left.\left.\left.+2\left(\partial_{\tilde{\ell}} \tilde{g}^{\tilde{A} \tilde{\ell}}\right) \partial_{\tilde{\ell}} \xi^{\tilde{B}}+2 \partial_{\tilde{\ell}} \tilde{g}^{\tilde{A} \tilde{A} \tilde{B}}\right)_{: \tilde{C}} \partial^{\tilde{u}} \partial_{\tilde{\ell}} \xi^{\tilde{B}}-\left(\partial_{\tilde{u}} \partial_{\tilde{\ell}} \tilde{g}^{\tilde{A} \tilde{B}}\right) \xi^{\tilde{u}}\right\}\left.\right|_{\mathscr{I}+} \tilde{g}^{\tilde{A} \tilde{B}}\right) \xi^{\tilde{C}}: \tilde{C}+2\left(\partial_{\tilde{\ell}} \xi^{\tilde{B}}\right)^{\tilde{A}}
\end{aligned}
$$

Here the $\partial_{\tilde{\ell}} \xi^{\tilde{A}}$ derivative of the Killing field is supplied from 5.15 . Using the properties of $\left.\xi^{\alpha}\right|_{\mathscr{I}+}$ and $\left.\partial_{\tilde{\ell}} \tilde{g}^{\tilde{A} \tilde{\ell}}\right|_{\mathscr{I}+}$, this reduces to

$$
\begin{aligned}
\mathcal{X} & =\frac{1}{4} Q_{\tilde{A}} Q_{\tilde{B}}\left\{\left(\partial_{\tilde{\ell}} \tilde{g}^{\tilde{A} \tilde{B}}\right) \xi^{\tilde{C}: \tilde{D}} Q_{[C} \bar{Q}_{D]}-\left(\partial_{\tilde{\ell}} \tilde{g}^{\tilde{A} \tilde{B}}\right)_{: \tilde{C}} \xi^{\tilde{C}}\right. \\
& \left.+2\left(\partial_{\tilde{\ell}} \xi^{\tilde{B}}\right)^{: \tilde{A}}+\partial_{\tilde{u}}\left(2 \tilde{g}^{\tilde{A} \tilde{u}} \partial_{\tilde{\ell}} \xi^{\tilde{B}}-\xi^{\tilde{u}} \partial_{\tilde{\ell}} \tilde{g}^{\tilde{A} \tilde{B}}\right)\right\}\left.\right|_{\mathscr{I}+}
\end{aligned}
$$

These expressions simplify for specific BMS symmetries.

For a supertranslation $\xi^{\tilde{u}}=\alpha\left(\tilde{x}^{A}\right), f^{\tilde{A}}=0$ and we have

$$
\left.\frac{\partial \xi^{\tilde{A}}}{\partial \tilde{\ell}}\right|_{\mathscr{I}^{+}}=\left.\left[\alpha \frac{\partial \tilde{g}^{\tilde{u} \tilde{A}}}{\partial \tilde{u}}-\alpha^{: \tilde{A}}\right]\right|_{\mathscr{I}^{+}},
$$

with the result that

$$
\mathcal{X}=\alpha \partial_{\tilde{u}} h-\frac{1}{2} Q_{\tilde{A}} Q_{\tilde{B}} \alpha^{: \tilde{A} \tilde{B}}
$$

For the translations, for which $\alpha\left(\tilde{x}^{A}\right)$ is an $\ell=0$ or $\ell=1$ spherical harmonic, $Q^{\tilde{A}} Q^{\tilde{B}} \alpha_{: \tilde{A} \tilde{B}}=0$. Since $\partial_{\tilde{u}} h=N$, we have

$$
\mathcal{X}=\alpha N
$$

For the rotations, $\alpha=0$ and $f^{\tilde{A}}=\epsilon^{\tilde{A} \tilde{B}} \Phi_{: \tilde{B}}$, where $\Phi$ is an $\ell=1$ harmonic satisfying $\Phi_{: \tilde{A}}^{\tilde{A}}=-2 \Phi$ with $\epsilon^{\tilde{A} \tilde{B}}=i Q^{[\tilde{A}} Q^{\tilde{B}]}, \epsilon^{\tilde{A} \tilde{C}} \epsilon_{\tilde{B} \tilde{C}}=\delta_{\tilde{B}}^{\tilde{A}}$. As a result, $f^{\tilde{A}: \tilde{B}}=$ 
$-\Phi \epsilon^{\tilde{A} \tilde{B}}$, and

$$
\left.\partial_{\tilde{\ell}} \xi^{\tilde{A}}\right|_{\mathscr{I}+}=\left.\left\{-g^{\tilde{u} \tilde{B}} f^{\tilde{A}}: \tilde{B}+f^{\tilde{B}} g^{\tilde{u} \tilde{A}}: \tilde{B}\right\}\right|_{\mathscr{I}+} .
$$

We define a strain tensor according to

$$
h^{\tilde{A} \tilde{B}}=\left.\frac{1}{4}\left\{-\partial_{\tilde{\ell}} \tilde{g}^{\tilde{A} \tilde{B}}+2 \tilde{g}^{\tilde{A} \tilde{u}} \partial_{\tilde{u}} \tilde{g}^{\tilde{u} \tilde{B}}+2 \tilde{g}^{\tilde{u}(\tilde{A}: \tilde{B})}\right\}\right|_{\mathscr{I}+},
$$

so that $Q_{\tilde{A}} Q_{\tilde{B}} h^{A B}=h$. Then a straightforward calculation gives

$$
\begin{aligned}
\mathcal{X} & =-2 i \Phi h+Q_{\tilde{A}} Q_{\tilde{B}} h^{\tilde{A} \tilde{B}}: \tilde{C} f^{\tilde{C}} \\
& +\left.Q_{\tilde{A}} Q_{\tilde{B}}\left\{f^{\tilde{C}} g_{: \tilde{B} \tilde{u}}^{\tilde{A}}-f^{\tilde{C}} g^{\tilde{B} \tilde{u}: \tilde{A}}: \tilde{C}+g^{\tilde{A} \tilde{u}} f^{\tilde{B}}\right\}\right|_{\mathscr{I}+}
\end{aligned}
$$

But the second line vanishes due to the identity

$$
V_{B: C A}-V_{B: A C}=Q_{A B} V_{C}-Q_{B C} V_{A}
$$

for the commutator of covariant derivatives with respect to the unit sphere metric $Q_{A B}$. Thus, for a rotation,

$$
\mathcal{X}=-2 i \Phi h+Q_{\tilde{A}} Q_{\tilde{B}} h^{\tilde{A} \tilde{B}}: \tilde{C} f^{\tilde{C}} .
$$

For the boosts, $\alpha=0, f^{\tilde{A}}=\Gamma^{: \tilde{A}}$, where $\Gamma^{: \tilde{A} \tilde{B}}=-\Gamma Q^{\tilde{A} \tilde{B}}, \Gamma^{: \tilde{A}}: \tilde{A}=-2 \Gamma, \xi^{\tilde{u}}=-u \Gamma$ and

$$
\left.\partial_{\tilde{\ell}} \xi^{\tilde{A}}\right|_{\mathscr{I}+}=\left.\left\{-\tilde{u} \Gamma \partial_{\tilde{u}} g^{\tilde{u} \tilde{A}}+\Gamma^{: \tilde{B}} g^{\tilde{u} \tilde{A}}: \tilde{B}+\tilde{u} \Gamma^{: \tilde{A}}\right\}\right|_{\mathscr{I}+} .
$$

Then by the analogous calculation leading to (5.44)

$$
\mathcal{X}=-\Gamma \partial_{\tilde{u}}(\tilde{u} h)+Q_{\tilde{A}} Q_{\tilde{B}} h^{\tilde{A} \tilde{B}}: \tilde{C}^{\Gamma^{: \tilde{C}}} .
$$

\section{Results}

Our results are based upon the same generic precessing binary black hole run taken from Taylor et al. [45], which was also used in [21] to calibrate the SpEC characteristic 
code and in 22] to compare waveform extraction using the SpEC and Pitt null codes using the gauge invariant version of the of the news function computed in computational coordinates. The physical parameters are mass ratio $q=3$, black hole dimensionless spins $\chi_{1}=(0.7,0,0.7) / \sqrt{2}$ and $\chi_{2}=(-0.3,0,0.3) / \sqrt{2}$, number of orbits 26 , total time $T=7509 M$, initial eccentricity $10^{-3}$, initial frequency $\omega_{i n i}=0.032 / M$ and extraction radius $R=100 M$, where $M$ is the total mass of the black holes.

Extraction was carried out at the three different resolutions elaborated in Table 1 to assess convergence.

\begin{tabular}{|r|ccc|}
\hline Run & Low & Med & High \\
\hline$N_{r}$ & 10 & 12 & 14 \\
$L$ & 12 & 14 & 17 \\
$\Delta t / M$ & 1.0 & $0.666 \ldots$ & 0.5 \\
\hline
\end{tabular}

Table 1: Resolution parameters used for code convergence comparisons, with time steps $\Delta t . N_{r}$ represents the radial grid sizes. The SpEC code has $2 L^{2}$ total angular grid points.

\subsection{Verification of strain, news, and radiative Weyl component $\Psi$}

Here we show that the news function computed using the inertial coordinate algorithm (inertial news) agrees with the gauge independent news (gauge-free news) computed in [22] using the computational coordinates. We also show that strain and and $\Psi$ (corresponding to the Newman-Penrose component $\bar{\psi}_{4}^{0}$ ) are consistent with the computation of the news function.

Comparison of the relative error $E_{\text {rel }}$ between dataset $A$ and dataset $B$ is computed according to

$$
E_{r e l}=\log _{10}\left(\frac{|A-B|}{|B|}\right)
$$


where in the convergence tests $B$ is the highest resolution dataset. For the strain, news function and radiative Weyl component $\Psi$, the real parts of the $(\ell, m)=(2,2)$ spherical harmonic modes are compared.

Spatial convergence is at an exponential rate expected of a spectral code, while time convergence is $4^{\text {th }}$ order. The convergence test shows which error source dominates the simulation. Given an increase in resolution by some constant factor, spectral convergence results in reducing the error by a constant factor on a log scale. In contrast, high-order polynomial convergence such as $E \propto \Delta t^{4}$ will yield logarithmic convergence on a $\log$ scale under the same increase in resolution. For these simulations, $4^{\text {th }}$ order time convergent error dominates over the spatial spectral error.

In Fig. 3, we computed the news function in two different ways at the three different resolutions outlined in Table 1, for a total of six datasets. The inertial news (computed directly in the inertial coordinate system) is compared with the high resolution results for the gauge-free news (computed first in the computational coordinates, as in [22]). Both versions of the news agree and display $4^{\text {th }}$ order convergence.

Similarly, we computed the error in the strain by comparing adjacent resolutions. The strain remains convergent over the entire run, as shown in Fig. 4. With only a $20 \%$ increase in resolution, the error in the medium resolution run decreases by $85 \%$, consistent with the underlying spatial spectral method and the $4^{\text {th }}$ order time integrator.

The higher time derivatives in $\Psi$ make it more sensitive to the numerical noise produced by the junk radiation in the initial phase of the run. After this initial period, the error in $\Psi$ displays the same rate of convergence as the news and strain, 


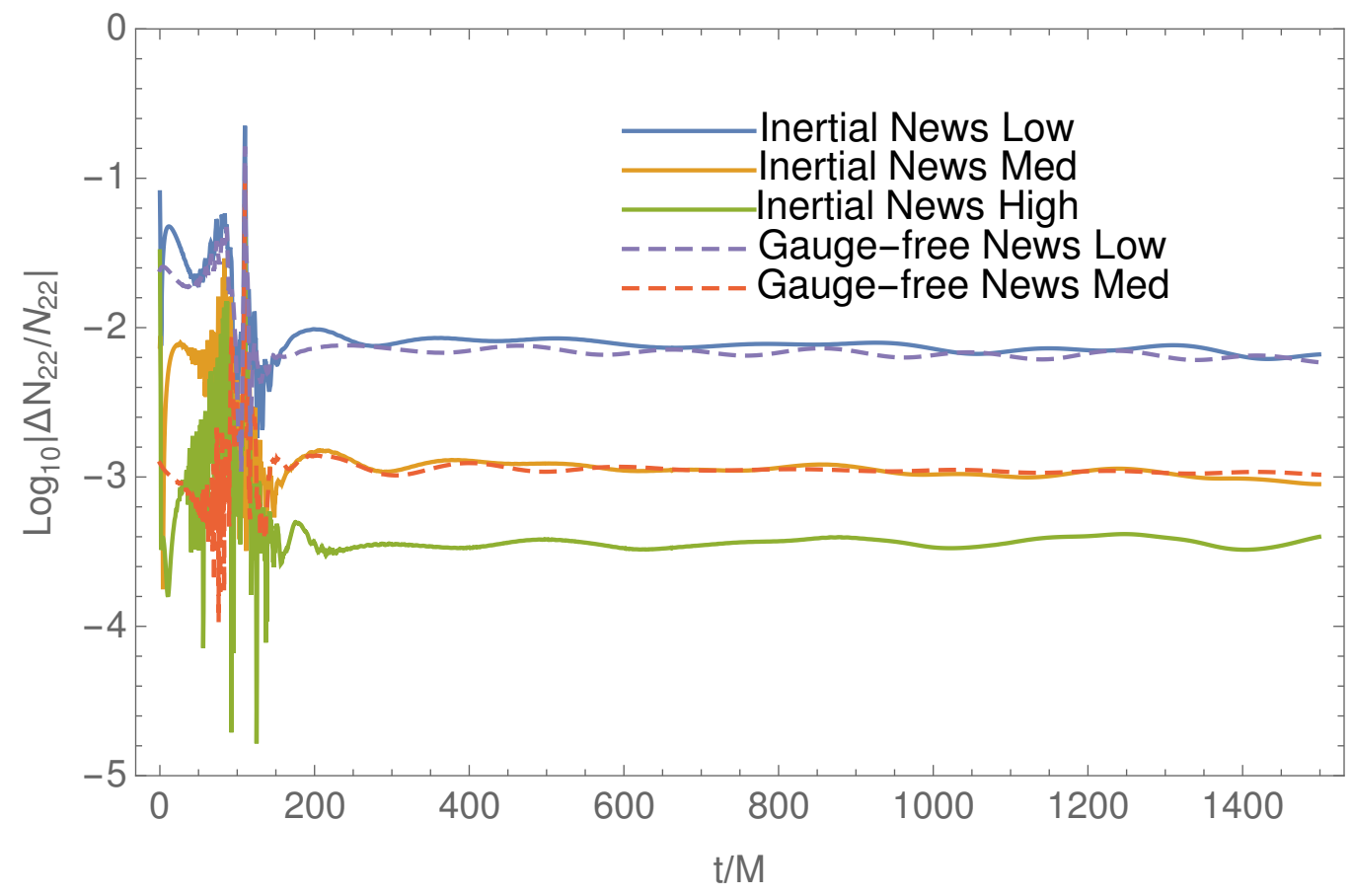

Figure 3: $4^{\text {th }}$ order convergence of the news function computed two different ways. Solid lines are the error in the new, inertial coordinates computation. Dashed lines are error in the gauge-free news computed in computational coordinates. Here, error is computed with reference to the highest resolution grid, showing both self convergence and agreement between the two methods.

as shown in Fig. 5 .

Finally, we verify the inertial frame relationships $\Psi=N_{, \tilde{u}}=h, \tilde{u} \tilde{u}$. Computed in the highest resolution inertial domain, these quantities agree, as shown in Fig. 6 .

\subsection{Flux}

In the inertial frame at $\mathscr{I}^{+}$, the asymptotic Killing vectors which generate the BMS group have the form 4.1 , i.e.

$$
\left.\xi^{\tilde{\rho}} \partial_{\tilde{\rho}}\right|_{\mathscr{I}^{+}}=\left(\alpha\left(\tilde{x}^{A}\right)+\frac{1}{2} \tilde{u} f_{: \tilde{A}}^{\tilde{A}}\right) \partial_{\tilde{u}}+f^{\tilde{A}} \partial_{\tilde{A}}
$$




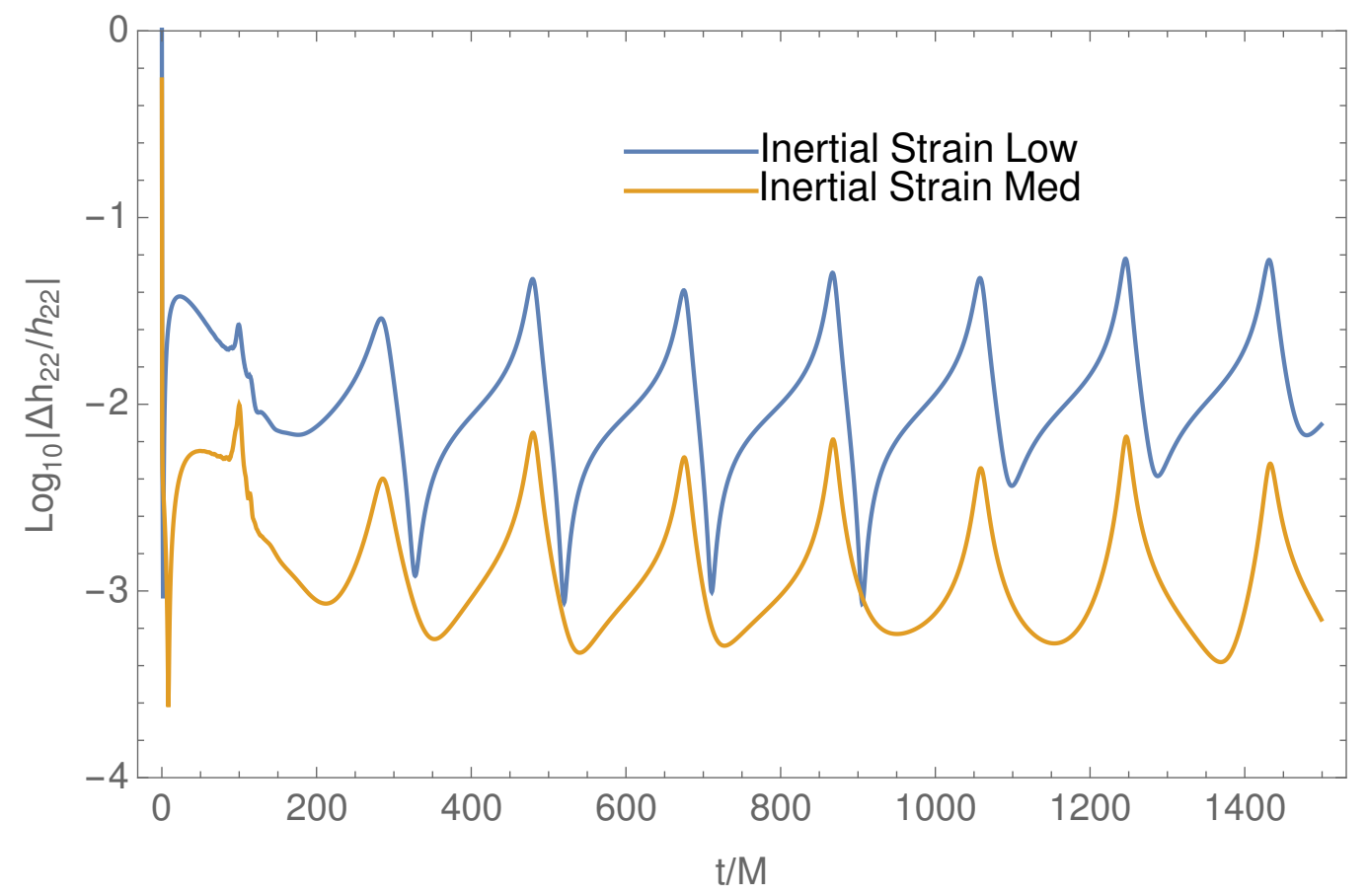

Figure 4: Here, we compare error in the low and medium resolution calculations of the strain computed in the inertial coordinates (inertial strain) by comparing them to the medium and high resolution runs, respectively. Comparison between the errors in the medium and low resolution runs is consistent with the $4^{\text {th }}$ order convergence arising from the time integrator.

where $f^{\tilde{A}}\left(\tilde{x}^{B}\right)$ is a conformal killing vector for the unit sphere metric. Here we consider the fluxes corresponding to the BMS generators for the time translation $T_{u}$, the three spatial translations $\left(T_{x}, T_{y}, T_{z}\right)$, the three rotations $\left(R_{x}, R_{y}, R_{z}\right)$, and the three boosts $\left(B_{x}, B_{y}, B_{z}\right)$, with respect to the corresponding axes of the asymptotic inertial frame, as well as a sample supertranslation (ST), totaling 11 asymptotic symmetries.

We compute the energy flux by calculating the absolute square $|N|^{2}$ of the news function. For the remaining flux calculations, we first use (5.34) to compute the flux rate of change and then carry out a retarded inertial time integral. As a result, the numerical noise in $\Psi$ during the initial phase of junk radiation (see Fig. 5 ) introduces some non-convergent error. Consequently, although the plots of the time dependence 


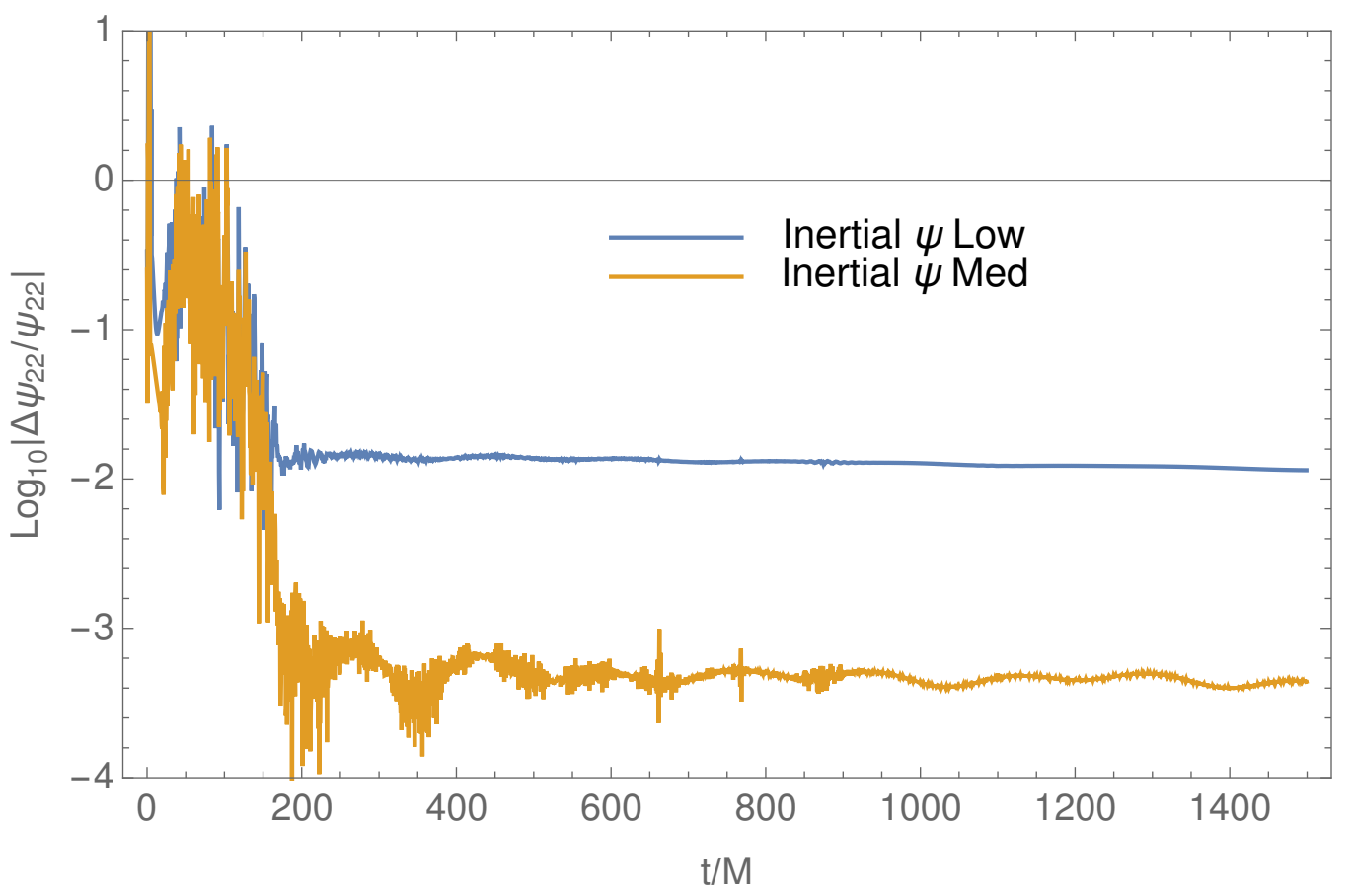

Figure 5: The errors in the inertial coordinate computation of $\Psi$ for low and medium resolution runs are compared with the medium and high resolution runs, respectively. The results confirm $4^{\text {th }}$ order convergence following the initial phase of junk radiation.

of the fluxes show good agreement for the three resolutions, convergence of the error is not as clean as for the energy flux computed directly from the news function. Therefore, for the purpose of convergence studies, we concentrate on the error in the retarded time derivative of the flux, although both rates of convergence are shown for completeness.

In all cases, we plot the waveform and convergence of the strongest mode. Part (a) of each plot is a spherical representation of the functional form of the corresponding BMS generator. Spatial components are shown with vector arrows, while the time component is demarcated with a color gradient.

6.2.1. Energy and momentum flux The time translation is described by the BMS generator $\xi_{[T u]}^{\alpha}$ with components $\alpha_{T u}=1, f^{A}=0$, corresponding to an $\ell=0$ spherical 


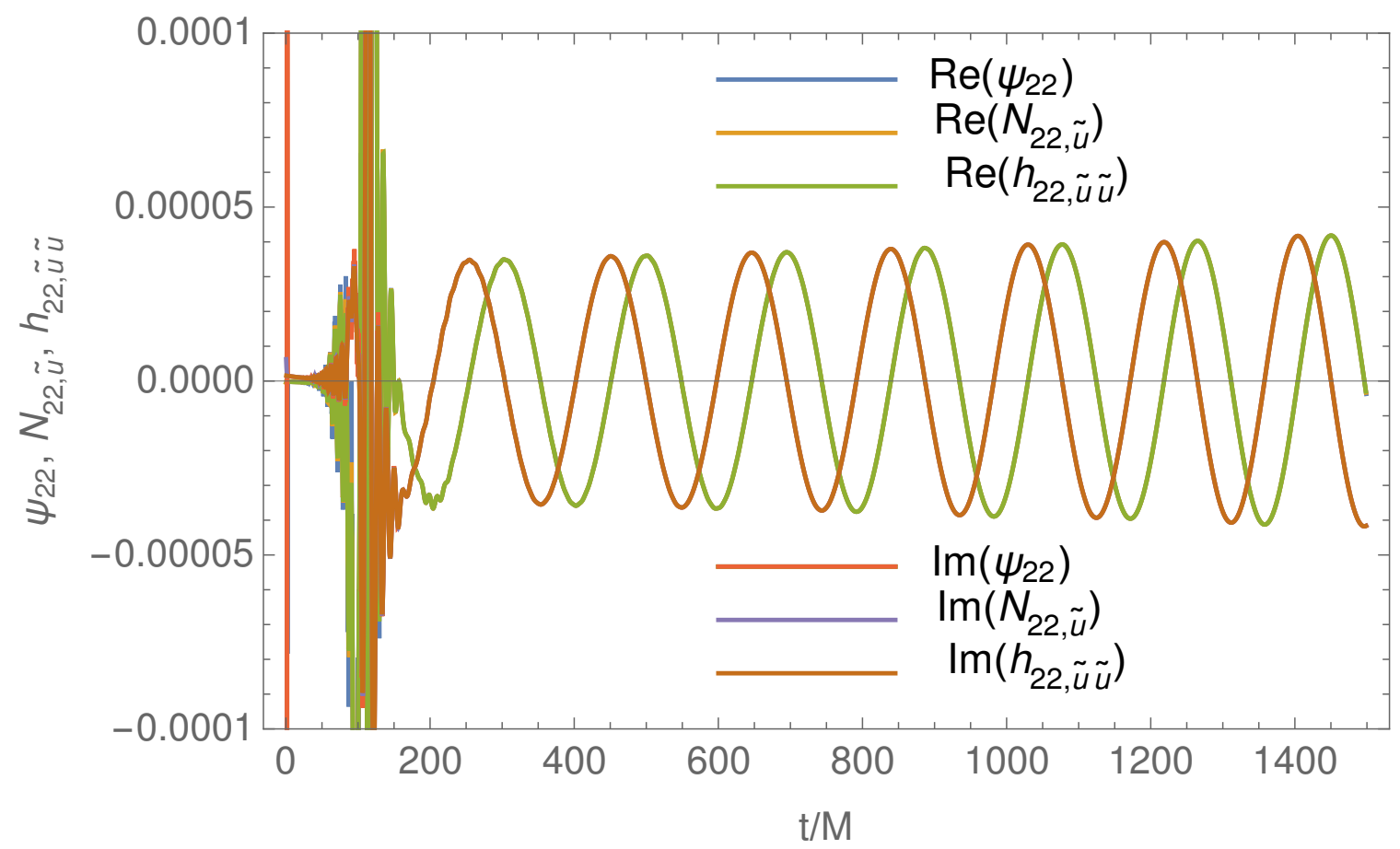

Figure 6: Comparison of $\Psi=N, \tilde{u}=h, \tilde{u} \tilde{u}$ (real and imaginary parts). Following the initial phase of junk radiation, the data overlap completely, hiding $\Psi$ and $N, \tilde{u}$ behind $h, \tilde{u} \tilde{u}$.

harmonic. Figure 7 shows the form of the generator $(7 \mathrm{a})$, its associated flux $(7 \mathrm{~b})$, the flux convergence $(7 \mathrm{c})$ and the stronger convergence of the inertial time derivative of the flux $(7 \mathrm{~d})$.

The three momentum fluxes are derived from the three spatial translations, described by the BMS generators $\xi_{[T x]}^{\alpha}, \xi_{[T y]}^{\alpha}$ and $\xi_{[T z]}^{\alpha}$, with $f^{A}=0$ and $\alpha$ constructed from $l=1$ spherical harmonics,

$$
\alpha_{[T x]}=\sin \tilde{\theta} \cos \tilde{\phi}, \quad \alpha_{[T y]}=\sin \tilde{\theta} \sin \tilde{\phi}, \quad \alpha_{[T z]}=\cos \tilde{\theta}
$$

corresponding to the axes of the asymptotic inertial frame. The corresponding momentum fluxes $F_{T x}, F_{T y}$ and $F_{T z}$ can also be obtained directly from the energy flux by weighting it with the corresponding $\ell=1$ harmonics,

$$
F_{T x}=\sin \tilde{\theta} \cos \tilde{\phi}|N|^{2}, \quad F_{T y}=\sin \tilde{\theta} \sin \tilde{\phi}|N|^{2}, \quad F_{T z}=\cos \tilde{\theta}|N|^{2},
$$


(a)

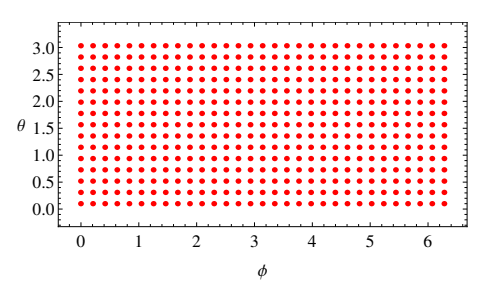

(c)

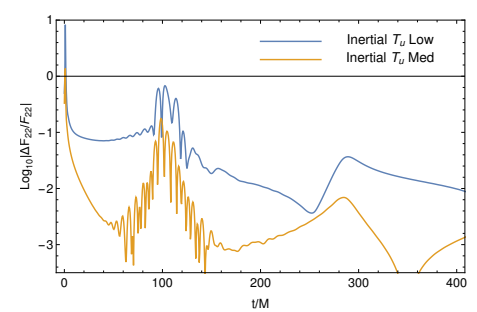

(b)

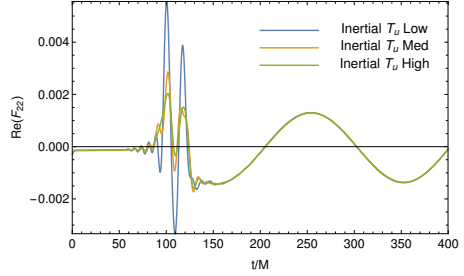

(d)

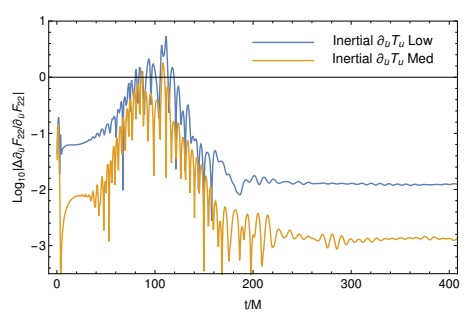

Figure 7: 7a) The $\tilde{u}$ component of $\xi_{T u}=1$ is uniform everywhere on the sphere and has no angular component. (7b) The $(\ell=2, m=2)$ spherical harmonic component of the flux, $F_{T u}=|N|^{2}$. (7c) Convergence of the flux is partially compromised by junk radiation, while the inertial time derivative of the flux $\sqrt{7 \mathrm{~d}}$ ) shows the appropriate $4^{\text {th }}$ order convergence following the junk phase.

in which case the clean $4^{\text {th }}$ order convergence obtained for the energy flux $|N|^{2}$ would also result following the initial period of junk radiation.

Alternatively, these momentum fluxes can be obtained by a retarded time integral, e.g. $\quad F_{T z}=\int \dot{F}_{T z} d u$. However, although the strain and energy flux both have dominant components in the $(\ell=2, m=2)$ mode, the nonlinear effect of multiplication by an $\ell=1$ harmonic shifts the momentum fluxes into other modes. For this reason, we plot the $(\ell=3, m=2)$ mode.

The $z$-component of momentum flux $F_{T z}$ obtained this way is shown in Fig. $8 \mathrm{~b}$. Convergence of the flux is shown in Fig. 8c and its inertial time derivative in Fig. 8d, 
(a)

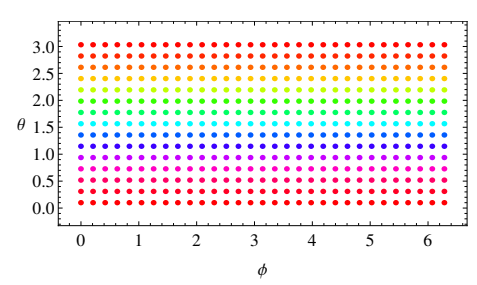

(c)

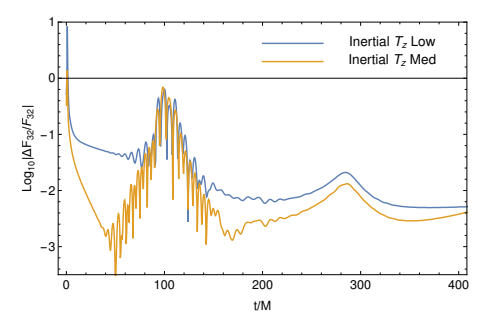

(b)

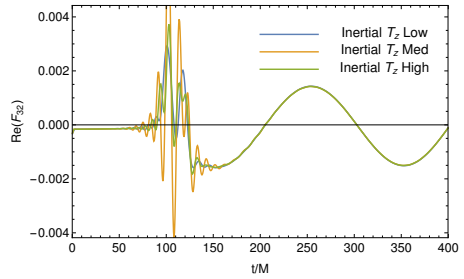

(d)

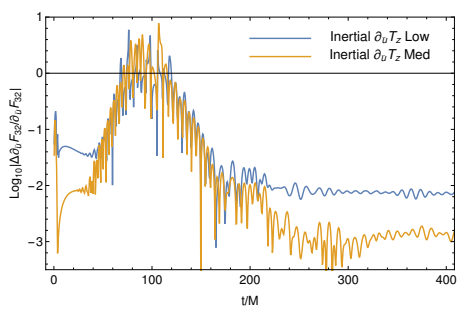

Figure 8: (8a) The $\tilde{u}$ component of $\xi_{T z}$ shifts from pole to pole, illustrating a translation in the $z$-direction. (8b) The $(\ell=3, m=2)$ spherical harmonic component of the flux. (8c) Convergence of the flux is partially compromised by junk radiation, while the inertial time derivative of the flux $8 \mathrm{~d}$ ) shows the appropriate $4^{\text {th }}$ order convergence following the junk phase.

The $(\ell=3, m=3)$ mode of the $x$-component of the momentum flux $F_{T x}$ is shown in Fig. 9b. Convergence of the flux is shown in Fig. 9c and its inertial time derivative in Fig. 9d.

Similarly, the $(\ell=3, m=3)$ mode of the $y$-component of the momentum flux $F_{T y}$ is shown in Fig. 10b. Convergence of the flux is shown in Fig. 10c and its inertial time derivative in Fig. 10d,

6.2.2. Rotations The three spatial rotations with respect to the inertial axes are described by the BMS generators $\xi_{[R x]}^{\alpha}, \xi_{[R y]}^{\alpha}$ and $\xi_{[R z]}^{\alpha}$ with $\alpha=0$ and $f^{\tilde{A}}=\epsilon^{\tilde{A} \tilde{B}} \Phi_{: \tilde{B}}$, 
(a)

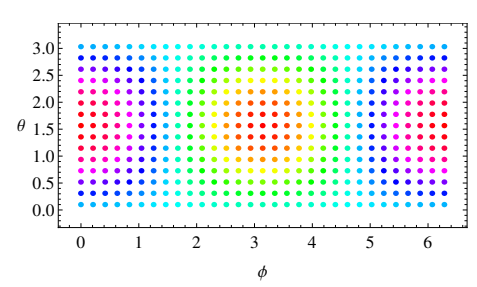

(c)

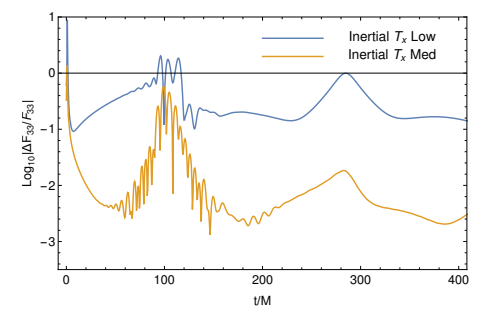

(b)

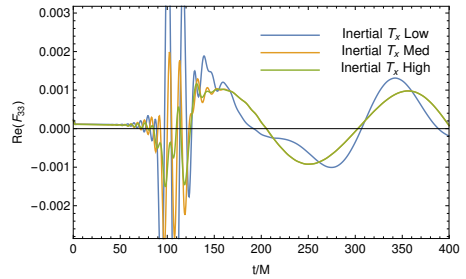

(d)

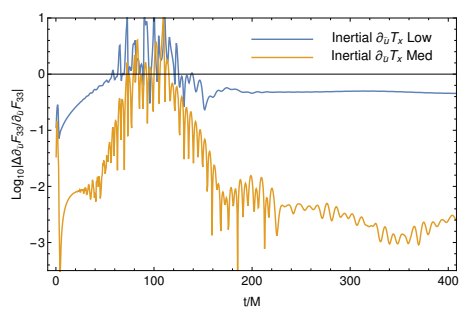

Figure 9: 9a The $\tilde{u}$ component of $\xi_{T x}$ shifts with $\phi$, showing a translation in the $x$ $(\phi=0)$ direction. (9b) The $(\ell=3, m=3)$ spherical harmonic component of the flux. 9c Convergence of the flux is partially compromised by junk radiation, while the inertial time derivative of the flux $9 \mathrm{~d}$ ) shows the appropriate $4^{\text {th }}$ order convergence following the junk phase.

where $\Phi$ is constructed from $\ell=1$ spherical harmonics. A rotation $R_{z}$ about the $z$ axis corresponds to the spherical harmonic $\Phi=\cos \tilde{\theta}$, so that $f^{\tilde{A}}=(0,1)$. Most of the motion of the orbiting black holes is about this axis, so we expect to see a greater flux of the corresponding $z$-component of angular momentum $F_{R z}$. The $(\ell=2, m=2)$ mode of the $z$ component of the angular momentum flux $F_{R z}$ is shown in Fig. $11 \mathrm{~b}$, Convergence of the flux is shown in Fig. $11 \mathrm{c}$ and its inertial time derivative in Fig. $11 \mathrm{~d}$.

A rotation $R_{x}$ about the $x$-axis corresponds to the spherical harmonic $\Phi=$ $\sin \tilde{\theta} \cos \tilde{\phi}$ so that $f^{\tilde{A}}=(-\sin \tilde{\phi},-\cot \tilde{\theta} \cos \tilde{\phi})$. Similarly, a rotation $R_{y}$ about the $y$-axis corresponds to the spherical harmonic $\Phi=\sin \tilde{\theta} \sin \tilde{\phi}$ so that $f^{\tilde{A}}=$ 
(a)

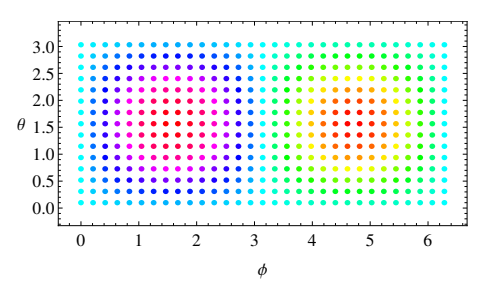

(c)

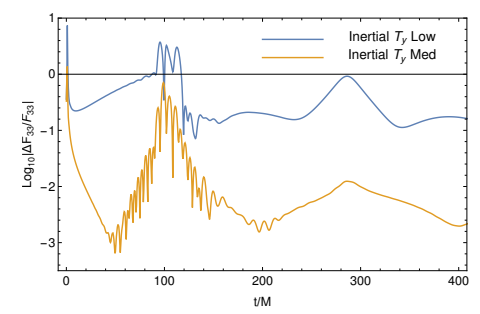

(b)

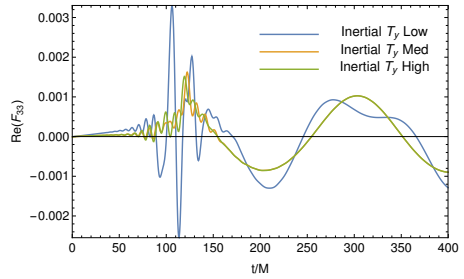

(d)

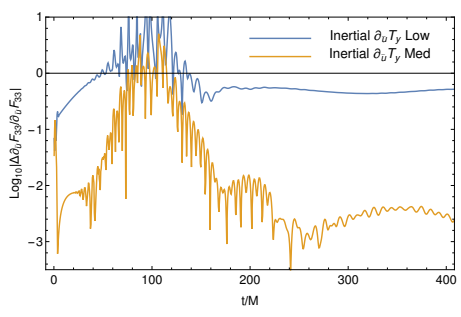

Figure 10: 10a The $\tilde{u}$ component of $\xi_{T y}$ shifts with $\phi$, showing a translation in the $y$ $(\phi=\pi / 2)$ direction. (10b) The $(\ell=3, m=3)$ spherical harmonic component of the flux. 10c Convergence of the flux is partially compromised by junk radiation, while the inertial time derivative of the flux $(10 \mathrm{~d})$ shows the appropriate $4^{\text {th }}$ order convergence following the junk phase.

$(\cos \tilde{\phi},-\cot \tilde{\theta} \sin \tilde{\phi})$

The $(\ell=2, m=1)$ modes of the $x$ and $y$-components of the angular momentum flux, $F_{R x}$ and $F_{R y}$, are shown in Fig. $12 \mathrm{~b}$ and Fig. $13 \mathrm{~b}$, respectively. Convergence of these fluxes is shown in Fig. $12 \mathrm{c}$ and Fig. 13c; and convergence of their inertial time derivative in Fig. 12d and Fig. 13d.

6.2.3. Boosts In addition to the rotations, the other transformations of the Lorentz group are the three boosts with BMS generators $\xi_{[B x]}^{\alpha}, \xi_{[B y]}^{\alpha}$ and $\xi_{[B z]}^{\alpha}$ for which $f_{: \tilde{A}}^{\tilde{A}} \neq 0$. For these boost generators, $\alpha=0, f^{\tilde{A}}=\Gamma^{: \tilde{A}}$, where $\Gamma$ consists of $\ell=1$ spherical 
(a)

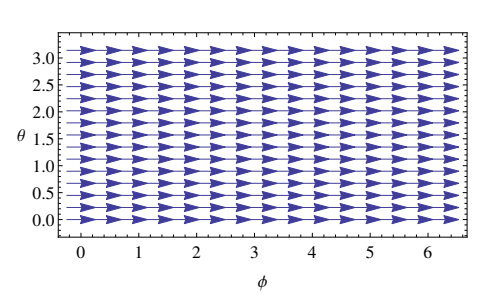

(c)

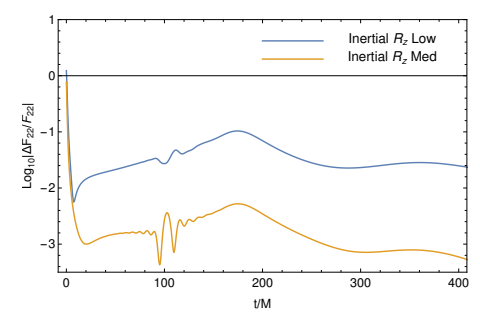

(b)

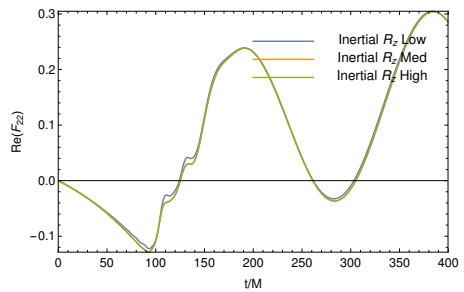

(d)

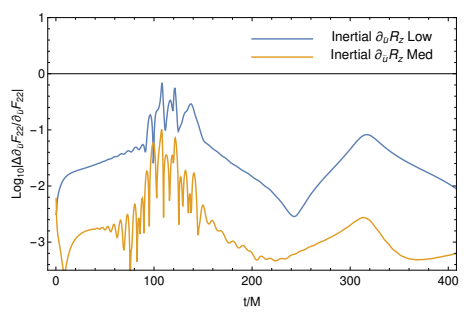

Figure 11: 11a) Vectors illustrate the nature of $\xi_{R z}$, a rotation about $z$. 11b The $(\ell=2, m=2)$ spherical harmonic component of the flux. (11c) Convergence of the flux is partially compromised by junk radiation, while the inertial time derivative of the flux (11d) shows the appropriate $4^{\text {th }}$ order convergence following the junk phase.

harmonics, so that $\Gamma_{: \tilde{A}}^{\tilde{A}}=-2 \Gamma$ and $\xi^{\tilde{u}}=-u \Gamma$. As a result, the boosts acquire a $\xi^{\tilde{u}}$ component with linear dependence on $\tilde{u}$, as well as $\ell=1$ angular dependence. The corresponding physical quantities describe the dipole moment of the system corresponding to the center-of-mass integrals in Lorentz covariant theories.

For a boost $B_{z}$ in the inertial $z$-direction, $\Gamma=\cos \tilde{\theta}$ so that $f^{\tilde{A}}=(-\sin \tilde{\theta}, 0)$ and $\xi_{[B z]}^{\tilde{u}}=-\tilde{u} \cos \tilde{\theta}$. The $(\ell=3, m=0)$ mode of the $z$-component of the boost flux $F_{B z}$ is shown in Fig. 14b. Convergence of the flux is shown in Fig. 14c and its inertial time derivative in Fig. 14d,

For a boost $B_{x}$ in the inertial $x$-direction, $\Gamma=\sin \tilde{\theta} \cos \tilde{\phi}$ so that $f^{\tilde{A}}=$ $(\cos \tilde{\theta} \cos \tilde{\phi},-\csc \tilde{\theta} \sin \tilde{\phi})$. Similarly, for a boost $B_{y}$ in the inertial $y$-direction, $\Gamma=$ 
(a)

(b)
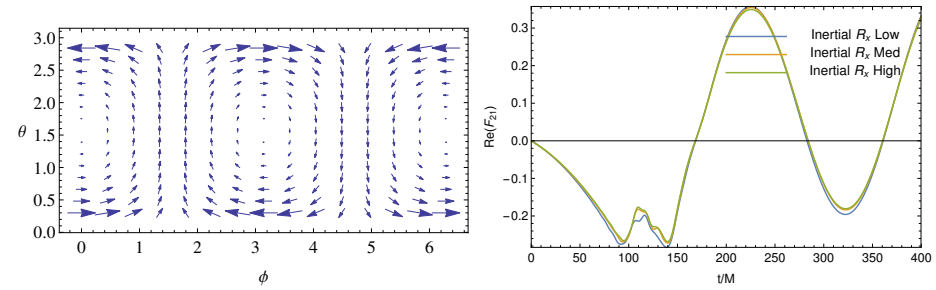

(c)

(d)
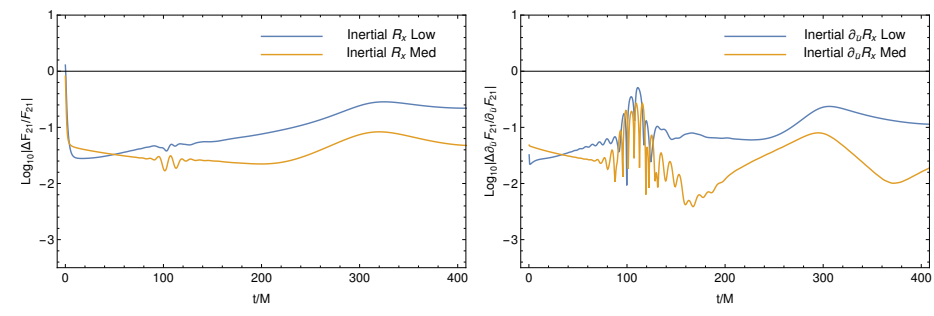

Figure 12: 12a) Vectors illustrate the nature of $\xi_{R x}$, a rotation about the $x$-axis $(\phi=0)$. (12b) The $(\ell=2, m=1)$ spherical harmonic component of the flux. (12c) Convergence of the flux is partially compromised by junk radiation, while the inertial time derivative of the flux $12 \mathrm{~d}$ shows the appropriate $4^{\text {th }}$ order convergence following the junk phase.

$\sin \tilde{\theta} \sin \tilde{\phi}$ so that $f^{\tilde{A}}=(\cos \tilde{\theta} \sin \tilde{\phi}, \csc \tilde{\theta} \cos \tilde{\phi})$. The $(\ell=3, m=3)$ mode of the $x$ and $y$-components of the boost flux, $F_{B x}$ and $F_{B y}$, is shown in Fig. $15 \mathrm{~b}$ and Fig. $16 \mathrm{~b}$ respectively. Convergence of these fluxes is shown in Fig. 15c and Fig. 16c; and convergence of their inertial time derivative in Fig. 15d and Fig. 16d,

6.2.4. Supertranslations The BMS supertranslation generators $\xi_{[S T]}^{\tilde{\alpha}}$ are described by $f^{\tilde{A}}=0$, with $\alpha\left(\tilde{x}^{B}\right)$ constructed from spherical harmonics with $l>1$. This leads to an infinite set of transformations, which extend well beyond the limit of code resolution. Here we concentrate on the supertranslation corresponding to a $\left|Y_{22}\right|$ spherical harmonic, $\alpha_{S T}=\sin ^{2} \tilde{\theta} \cos 2 \tilde{\phi}$. 
(a)

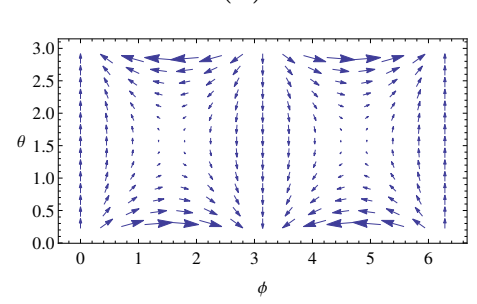

(c)

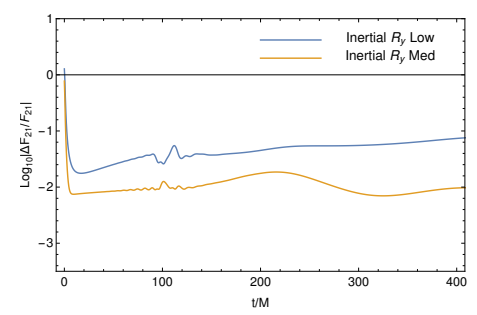

(b)

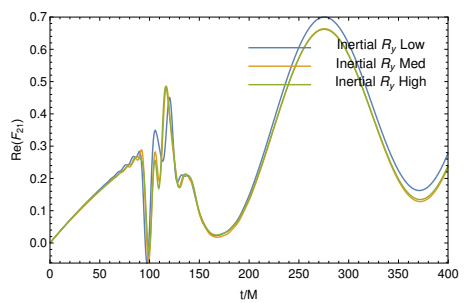

(d)

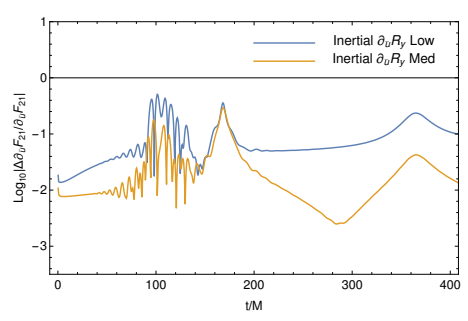

Figure 13: 13a) Vectors illustrate the nature of $\xi_{R y}$, a rotation about $y(\phi=\pi / 2)$. (13b) The $(\ell=2, m=1)$ spherical harmonic component of the flux. $13 \mathrm{c}$ Convergence of the flux is partially compromised by junk radiation, while the inertial time derivative of the flux 13d shows the appropriate $4^{\text {th }}$ order convergence following the junk phase.

The $(\ell=2, m=2)$ mode of the $\left|Y_{22}\right|$-derived supertranslation flux $F_{S T}$ is shown in Fig. 17b. Convergence of the flux is shown in Fig. $17 \mathrm{c}$ and its inertial time derivative in Fig. $17 \mathrm{~d}$,

\section{Conclusion}

In the context of Cauchy-characteristic evolution, we have developed the mathematical formalism for computing the gravitational radiation fluxes to $\mathscr{I}^{+}$ of energy-momentum, angular-momentum-dipole-moment and supermomentum, associated with the asymptotic symmetries of the BMS group. We have implemented 
(a)

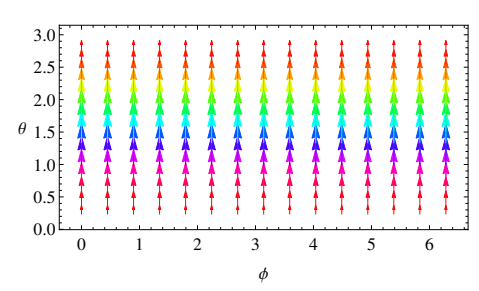

(c)

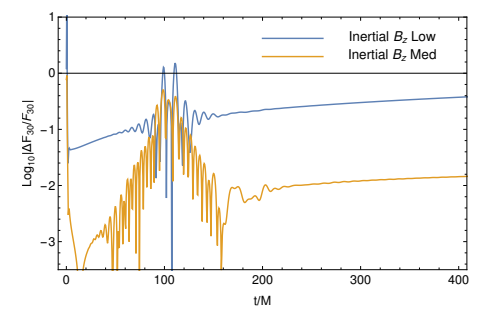

(b)

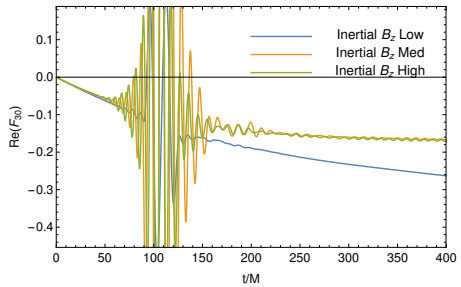

(d)

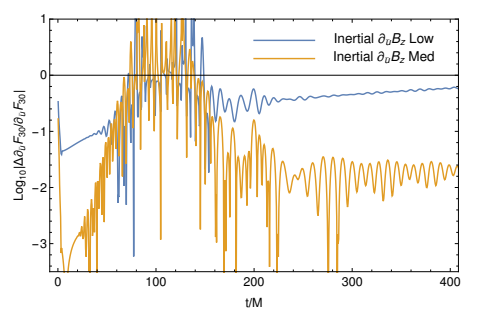

Figure 14: 14a Vectors illustrate the nature of $\xi_{B z}^{\tilde{A}}$, while colors illustrate the gradient of $\xi_{B z}^{\tilde{u}}$, illustrating a Lorentz boost $B z$ in the $z$-direction. $14 \mathrm{~b}$ The $(\ell=3, m=0)$ spherical harmonic component of the flux. 14c) Convergence of the flux is partially compromised by junk radiation, while the inertial time derivative of the flux $14 \mathrm{~d}$ shows the appropriate $4^{\text {th }}$ order convergence following the junk phase.

this algorithm as part of the Spectral Einstein Code (SpEC). The resulting code supplies a uniform computation of the radiation strain, news function, NewmanPenrose radiative $\psi_{4}^{0}$ curvature component and BMS fluxes in terms of inertial coordinates at $\mathscr{I}^{+}$. It is a stable, convergent, and highly efficient code for determining all the physical attributes of the gravitational radiation field.

Convergence tests were carried out based upon the simulation of a generic precessing binary black hole. These tests showed that the numerical accuracy was limited by the $4^{\text {th }}$ order time integrator, as opposed to the exponential convergence rate expected of the spatial spectral code. The main source of error arose from the 
(a)

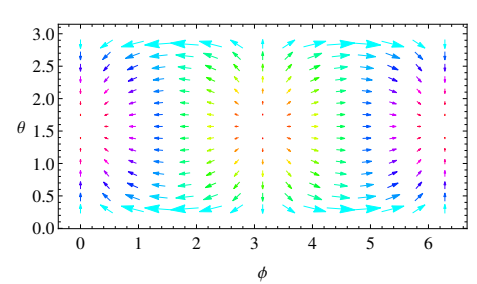

(c)

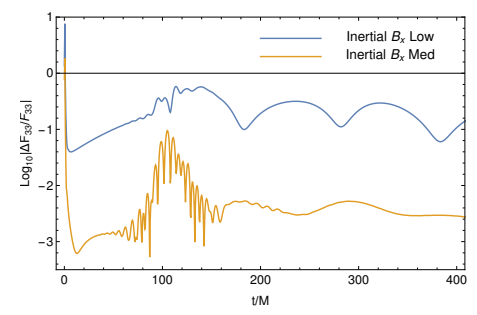

(b)

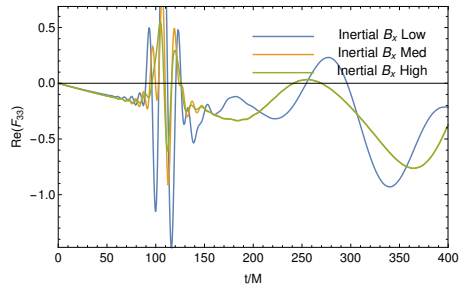

(d)

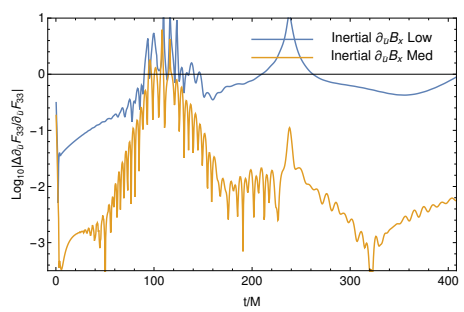

Figure 15: 15a Vectors illustrate the nature of $\xi_{B x}^{\tilde{A}}$, while colors illustrate the gradient of $\xi_{B x}^{\tilde{u}}$, illustrating a boost in the $x(\phi=0)$ direction. $15 \mathrm{~b}$ The $(\ell=3, m=3)$ spherical harmonic component of the flux. (15c) Convergence of the flux is partially compromised by junk radiation, while the inertial time derivative of the flux $15 \mathrm{~d}$ shows the appropriate $4^{\text {th }}$ order convergence following the junk phase.

artificial junk radiation introduced by the binary black hole initial data.

The accuracy for radiation strain, news function $N$ and $\psi_{4}^{0}$ were comparable to computations using a prior version of the SpEC characteristic code. The same was also found for the computation of energy-momentum flux, which is determined by the $\ell=0$ and $\ell=1$ components of $|N|^{2}$.

The computation of the angular momentum and supermomentum fluxes is more complicated than the energy-momentum flux. In addition, there are ambiguities in their underlying construction, which we base here upon the linkage integrals. However, these ambiguities are not as serious in the case of the retarded time 
(a)

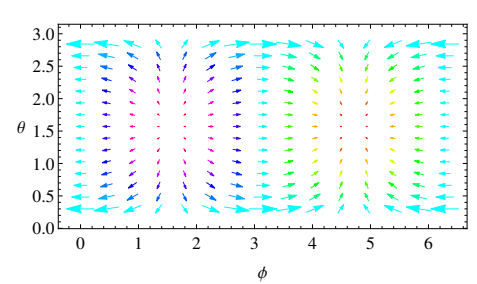

(c)

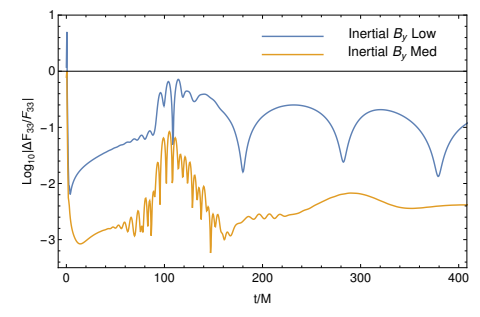

(b)

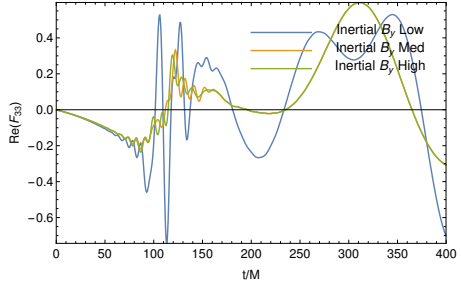

(d)

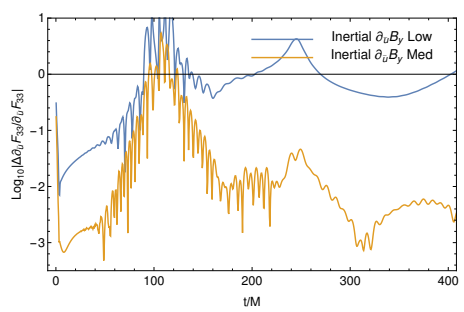

Figure 16: $16 \mathrm{a}$ Vectors illustrate the nature of $\xi_{B y}^{\tilde{A}}$, while colors illustrate the gradient of $\xi_{B y}^{\tilde{u}}$, illustrating a boost in the $y(\phi=\pi / 2)$ direction. $16 \mathrm{~b}$ The $(\ell=3, m=3)$ spherical harmonic component of the flux. (16c) Convergence of the flux is partially compromised by junk radiation, while the inertial time derivative of the flux $16 \mathrm{~d}$ ) shows the appropriate $4^{\text {th }}$ order convergence following the junk phase.

derivatives of the linkage flux $\dot{F}_{\xi}$, which only depend upon a product of $\psi_{4}^{0}$ with the choice of BMS generator, according to (5.34). An idealized strategy for studying, say, angular momentum would be to base its initial value either on the Wald-Zoupas Hamiltonian approach [34] in the infinite past retarded time $u \rightarrow-\infty$ or on its unambiguous definition at spatial infinity, where the initial flux should vanish. The dynamical properties of angular momentum can then be studied by retarded time integrals of $\dot{F}_{\xi}$. However, in practice this strategy would require binary black hole data devoid of junk radiation, which is not true at least for the generic precessing system simulated here. Following the the initial phase of junk radiation, tests of $\dot{F}_{\xi}$ 
(a)

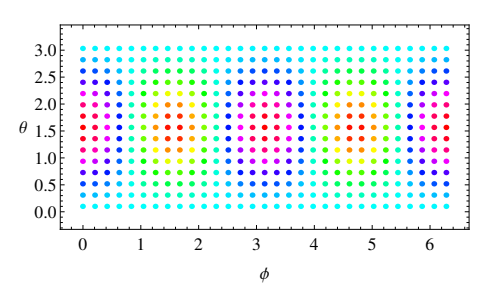

(c)

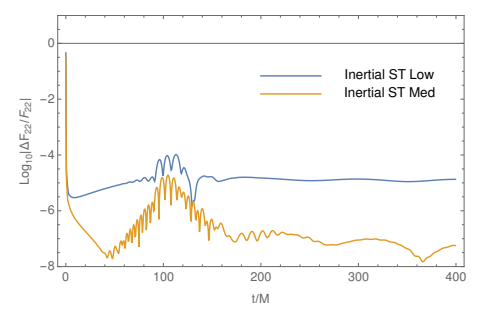

(b)

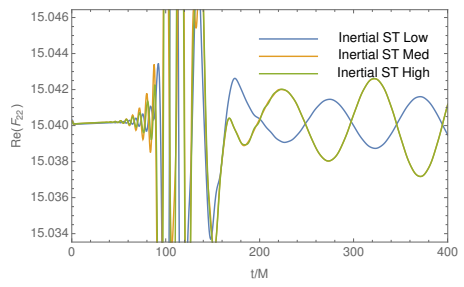

(d)

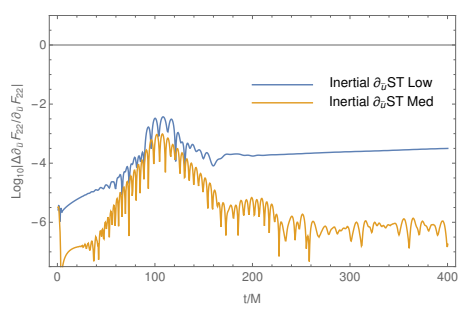

Figure 17: 17a A color gradient illustrates the nature of $\xi_{S T}^{\tilde{u}}$, a $\left|Y_{22}\right|$ supertranslation. (17b) The $(\ell=2, m=2)$ spherical harmonic component of the flux. 17c Convergence of the flux is partially compromised by junk radiation, while the inertial time derivative of the flux $17 \mathrm{~d}$ shows the appropriate $4^{\text {th }}$ order convergence following the junk phase.

showed clear $4^{\text {th }}$ order convergence. However, the high derivatives involved in the calculation of $\dot{F}_{\xi}$ magnify the effect of the junk radiation.

These considerations add to other important reasons to develop methods for obtaining binary black hole data which suppress junk radiation. In particular, this would allow application of our code to study the interesting question of how a supertranslation shift between the preferred Poincaré groups at $u= \pm \infty$ might affect angular momentum loss. 


\section{Acknowledgments}

We thank Nicholas Taylor for his generic precessing binary black hole run that we used to test and baseline code performance. We thank Mark Scheel, Yanbei Chen, and Christian Reisswig for their advice, support, and technical expertise. This research used the Spectral Einstein Code (SpEC)[46]. The Caltech cluster zwicky.cacr.caltech.edu is an essential resource for SpEC related research, supported by the Sherman Fairchild Foundation and by NSF award PHY-0960291. This research also used the Extreme Science and Engineering Discovery Environment (XSEDE) under grant TG-PHY990002. The UCSD cluster ccom-boom. ucsd.edu was used during code development. This project was supported by the Sherman Fairchild Foundation, and by NSF Grants PHY-1068881, AST-1333520, and CAREER Grant PHY-0956189 at Caltech. JW's research was supported by NSF grant PHY-1505965 to the University of Pittsburgh.

\section{References}

[1] B. P. A. et al. (LIGO Scientific Collaboration and V. Collaboration), "Observation of gravitational waves from a binary black hole merger," Phy. Rev. Lett. 116 (2016) 061102.

[2] S. J. Waldman, "The advanced ligo gravitational wave detector," Tech. Rep. LIGO-P0900115-v2, LIGO Project, 2011.

[3] T. Accadia, F. Acernese, F. Antonucci, P. Astone, G. Ballardin, et al., "Plans for the upgrade of the gravitational wave detector VIRGO: Advanced VIRGO," in Proceedings of the Twelfth Marcel Grossmann Meeting on General Relativity, T. Damour, R. T. Jantzen, and R. Ruffini, eds., pp. 1738-1742. 2009.

[4] LIGO Scientific Collaboration Collaboration, H. Grote, "The GEO 600 status," Class. Quantum Grav. 27 (2010) 084003

[5] K. Somiya and the KAGRA Collaboration, "Detector configuration of KAGRA-the japanese cryogenic gravitational-wave detector," Class. Quantum Grav. 29 no. 12, (2012) 124007. 
[6] W. Tichy and P. Marronetti, "Binary black hole mergers: Large kicks for generic spin orientations," Phys. Rev. D 76 (2007) 061502(R).

[7] C. O. Lousto and Y. Zlochower, "Hangup Kicks: Still Larger Recoils by Partial Spin/Orbit Alignment of Black-Hole Binaries," Phys. Rev. Lett. 107 (2011) 231102, arXiv:1108.2009 [gr-qc].

[8] J. A. González, U. Sperhake, B. Brügmann, M. Hannam, and S. Husa, "Maximum kick from nonspinning black-hole binary inspiral," Phys. Rev. Lett. 98 (2007) 091101, gr-qc/0610154

[9] M. Favata, S. A. Hughes, and D. E. Holz, "How black holes get their kicks: Gravitational radiation recoil revisited," Astrophys. J. 607 (2004) L5-L8.

[10] J. G. Baker, W. D. Boggs, J. Centrella, B. J. Kelly, S. T. McWilliams, M. C. Miller, and J. R. van Meter, "Modeling kicks from the merger of generic black-hole binaries," Astrophys. J. 682 (2008) L29, arXiv:0802.0416.

[11] J. Healy, F. Herrmann, I. Hinder, D. M. Shoemaker, P. Laguna, and R. A. Matzner, "Superkicks in hyperbolic encounters of binary black holes," Phys. Rev. Lett. 102 (2009) 041101, arXiv:0807.3292 [gr-qc].

[12] H. Bondi, M. G. J. van der Burg, and A. W. K. Metzner, "Gravitational waves in general relativity VII. Waves from axi-symmetric isolated systems," Proc. R. Soc. Lond. A 269 (1962) 21-52.

[13] R. K. Sachs, "Gravitational waves in general relativity. VIII. waves in asymptotically flat space-time," Proc. R. Soc. Lond. A 270 no. 1340, (October, 1962) 103-126. http://www.jstor.org/stable/2416200

[14] R. Penrose, "Asymptotic properties of fields and space-times," Phys. Rev. Lett. 10 no. 2, (1963) 66-68.

[15] J. Winicour, "Characteristic evolution and matching," Living Rev. Rel. 15 no. 2, (2012) . http://www.livingreviews.org/lrr-2012-2.

[16] L. A. Tamburino and J. H. Winicour, "Gravitational fields in finite and conformal Bondi frames," Phys. Rev. 150 (1966) 1039-1053. http://link.aps.org/doi/10.1103/PhysRev.150.1039.

[17] R. A. Isaacson, J. S. Welling, and J. Winicour, "Null cone computation of gravitational radiation," J. Math. Phys. 24 (1983) 1824. http://iopscience.iop.org/0264-9381/30/7/075017. 
[18] N. T. Bishop, R. Gomez, L. Lehner, M. Maharaj, and J. Winicour, "High-powered gravitational news," Phys. Rev. D56 (1997) 6298-6309, arXiv:gr-qc/9708065.

[19] M. C. Babiuc, N. T. Bishop, B. Szilágyi, and J. Winicour, "Strategies for the characteristic extraction of gravitational waveforms," Phys. Rev. D 79 (2009) 084011, arXiv:0808.0861.

[20] M. C. Babiuc, B. Szilágyi, J. Winicour, and Y. Zlochower, "A characteristic extraction tool for gravitational waveforms," Phys. Rev. D 84 (Aug, 2011) 044057, arXiv:1011.4223 [gr-qc]. http://link.aps.org/doi/10.1103/PhysRevD.84.044057.

[21] C. J. Handmer and B. Szilágyi, "Spectral characteristic evolution: A new algorithm for gravitational wave propagation," Classical and Quantum Gravity 32 (2015) 025008, arXiv:1406.7029.

[22] C. J. Handmer, B. Szilágyi, and J. Winicour, "Gauge invariant spectral characteristic extraction," arXiv:1502.06987.

[23] R. Geroch, Asymptotic Structure of Spacetime. Plenum, New York, 1995.

[24] L. B. Szabados, "Quasi-local energy-momentum and angular momentum in General Relativity: A review article," Living Rev. Rel. 12 no. 4, (2009) . http://www.livingreviews.org/lrr-2009-4

[25] A. Ashtekar and R. O. Hansen, "A unified treatment of null and spatial infinity in general relativity. i. universal structure, asymptotic symmetries, and conserved quantities at spatial infinity," J. Math. Phys. 19 (1978) 1542-1566.

[26] R. K. Sachs, "Asymptotic symmetries in gravitational theory," Phys. Rev. 128 (1962) $2851-2864$.

[27] E. T. Newman and R. Penrose, "Note on the Bondi-Metzner-Sachs group," J. Math. Phys. 7 (1966) 863-870, http://link.aip.org/link/?JMP/7/863/1

[28] J. Winicour, "Angular momentum in general relativity," in General Relativity and Gravitation, A. Held, ed., vol. 2, pp. 71-96. Plenum Press, New York, 1980.

[29] R. Geroch and J. Winicour, "Linkages in general relativity," J. Math. Phys. 22 (1981) 803.

[30] J. Winicour, "Global aspects of radiation memory," Classical and quantum gravity 31 no. 20, (2014) 205003

[31] J. Winicour, "Some total invariants of asymptotically flat space-times," J. Math. Phys. 9 no. $6,(1968) 861-867$.

[32] A. Komar, "Covariant conservation laws in general relativity," Phys. Rev. 113 (1959) 934-936 
[33] A. Ashtekar and M. Streubel, "Symplectic geometry of radiative modes and conserved quantities at null infinity," Proc. R. Soc. Lond. A 376 no. 1767, (1981) 585-607.

[34] R. Wald and A. Zoupas, "General definition of "conserved quantities" in general relativity and other theories of gravity," Phys. Rev. D 61 (2000) 084027.

[35] T. Dray and M. Streubel, "Angular momentum at null infinity," Classical and Quantum Gravity 1 no. 1, (1984) 15.

[36] A. Ashtekar and J. Winicour, "Linkages and hamiltonians at null infinity," Journal of Mathematical Physics 23 no. 12, (1982) 2410-2417.

[37] A. D. Helfer, "Angular momentum of isolated systems," General Relativity and Gravitation 39 no. 12 , (2007) 2125-2147.

[38] R. Penrose, "Quasi-local mass and angular momentum in general relativity," Proceedings of the Royal Society of London A 381 no. 1780, (1982) 53-63.

[39] A. I. Nesterov, "Quasigroups, asymptotic symmetries, and conservation laws in general relativity," Phys. Rev. D 56 no. 12, (1997) R7498.

[40] N. Bishop and S. Deshingkar, "New approach to calculating the news," Phys. Rev. D. 68 (2003) 024031.

[41] N. T. Bishop and C. Reisswig, "The gravitational wave strain in the characteristic formalism of numerical relativity," Gen. Rel. Grav. 46 (2014) 1843.

[42] M. Boyle, "Transformations of asymptotic gravitational-wave data," Phys. Rev. D 93 (2016) 084031.

[43] A. D. Helfer, "Estimating energy-momentum and angular momentum near null infinity," Phys. Rev. D 81 (2010) 084001.

[44] J. Winicour, "Newtonian gravity on the null cone," J. Math. Phys. 1193 (1983) .

[45] N. W. Taylor, M. Boyle, C. Reisswig, M. A. Scheel, T. Chu, L. E. Kidder, and B. Szilágyi, "Comparing gravitational waveform extrapolation to Cauchy-characteristic extraction in binary black hole simulations," Phys. Rev. D 88 (Dec, 2013) 124010, arXiv:1309.3605 [gr-qc], http://link.aps.org/doi/10.1103/PhysRevD.88.124010.

[46] A. H. Mroue, M. A. Scheel, B. Szilagyi, H. P. Pfeiffer, M. Boyle, D. A. Hemberger, L. E. Kidder, G. Lovelace, S. Ossokine, N. W. Taylor, A. Zenginoglu, L. T. Buchman, T. Chu, E. Foley, M. Giesler, R. Owen, and S. A. Teukolsky, "A catalog of 174 binary black hole simulations for gravitational wave astronomy," Phys. Rev. Lett. 111 (2013) 241104, 
arXiv:1304.6077 [gr-qc] 